\title{
Stable finite difference schemes for the magnetic induction equation with Hall effect
}

\section{Journal Article}

\section{Author(s):}

Corti, Pablo; Mishra, Siddhartha

Publication date:

2012-12

Permanent link:

https://doi.org/10.3929/ethz-b-000044294

\section{Rights / license:}

In Copyright - Non-Commercial Use Permitted

\section{Originally published in:}

BIT Numerical Mathematics 52(4), https://doi.org/10.1007/s10543-012-0383-3 


\title{
Stable finite difference schemes for the magnetic induction equation with Hall effect
}

\author{
Paolo Corti · Siddhartha Mishra
}

Received: 3 May 2011 / Accepted: 3 April 2012 / Published online: 9 May 2012

(C) Springer Science + Business Media B.V. 2012

\begin{abstract}
We consider a sub-model of the Hall-MHD equations: the so-called magnetic induction equations with Hall effect. These equations are non-linear and include third-order spatial and spatio-temporal mixed derivatives. We show that the energy of the solutions is bounded and design finite difference schemes that preserve the energy bounds for the continuous problem. We design both divergence preserving schemes and schemes with bounded divergence. We present a set of numerical experiments that demonstrate the robustness of the proposed schemes.
\end{abstract}

Keywords Finite difference methods $\cdot$ Stability and convergence of numerical methods

Mathematics Subject Classification 65M06 · 65M12

\section{Introduction}

Plasmas are increasingly becoming important in a variety of fields like astrophysics, solar physics, electrical and aerospace engineering [6]. Specific problems include the study of supernovas, accretion disks, waves in the solar atmosphere, magnetic confinement fusion, the design of plasma thrusters for spacecraft propulsion and of circuit breakers in the electrical power industry. It is standard to model plasmas as magnetized fluids with fluid motion shaping and in turn being shaped by magnetic

Communicated by Per Lötstedt.

P. Corti $(\bowtie) \cdot$ S. Mishra

Seminar For Applied Mathematics (SAM), ETH Zentrum, Rämistrasse 101, 8092 Zürich,

Switzerland

e-mail: paolo.corti@sam.math.ethz.ch

S. Mishra

e-mail: siddhartha.mishra@sam.math.ethz.ch 
fields. The base model for such interaction are the equations of Magneto-Hydro Dynamics (MHD):

$$
\begin{aligned}
\frac{\partial \rho}{\partial t} & =-\nabla \cdot(\rho \mathbf{u}), \\
\frac{\partial(\rho \mathbf{u})}{\partial t} & =-\nabla\left\{\rho \mathbf{u} \otimes \mathbf{u}+\left(p+\frac{|\mathbf{B}|^{2}}{2}\right) \mathbf{I}_{3 \times 3}-\mathbf{B} \otimes \mathbf{B}\right\}, \\
\frac{\partial \mathscr{E}}{\partial t} & =-\nabla\left\{\left(\mathscr{E}+p-\frac{|\mathbf{B}|^{2}}{2}\right) \mathbf{u}+\mathbf{E} \times \mathbf{B}\right\}, \\
\frac{d \mathbf{B}}{d t} & =-\nabla \times \mathbf{E} .
\end{aligned}
$$

Here $\rho, \mathbf{u}, p$ are the gas density, velocity and pressure respectively. $\mathbf{E}$ and $\mathbf{B}$ are the electric and magnetic fields. The total energy $\mathscr{E}$ is given by the equation of state:

$$
\mathscr{E}=\frac{p}{\gamma-1}+\frac{\rho|\mathbf{u}|^{2}}{2}+\frac{|\mathbf{B}|^{2}}{2} .
$$

Here, $\gamma$ is the gas constant. Equations from (1.1) to (1.3) represent the conservation of mass, momentum and energy and (1.4) describes the Maxwell's equations for the evolution of the magnetic field.

Applying the divergence operator to Maxwell's equations results in,

$$
\frac{d(\nabla \cdot \mathbf{B})}{d t}=0 .
$$

Furthermore, magnetic monopoles have not been observed in nature. The above equation implies that if the divergence of the initial magnetic field is zero, then solutions of the MHD equations also obey the divergence constraint:

$$
\nabla \cdot \mathbf{B}=0 .
$$

We need to describe the electric field in (1.4) to complete the MHD equations. Different choices for the electric field (Ohm's law) lead to different MHD models. The most popular MHD model are the ideal MHD equations where the electric field is given by

$$
\mathbf{E}=-\mathbf{u} \times \mathbf{B} .
$$

The ideal MHD equations are extremely successful in several applications, see [1] for an overview and [4] for a recent application in solar physics. However a major limitation of the ideal MHD equations is the requirement that the magnetic field lines are frozen into the fluid. In many interesting applications in both astrophysics and engineering, we observe magnetic reconnection, i.e., the magnetic topology changes during the flow [11]. In order to induce reconnection, a possible mechanism is magnetic resistivity resulting in the Ohm's law:

$$
\mathbf{E}=-\mathbf{u} \times \mathbf{B}+\eta \mathbf{J} .
$$


Here $\mathbf{J}$ is the current density and $\eta$ is the resistivity parameter. The resulting equations are termed the resistive MHD equations. However, the resistive MHD equations do not suffice in modeling fast magnetic reconnection.

A more effective alternative is to include the Hall effect $[11,15]$. The resulting Ohm's law is

$$
\mathbf{E}=-\mathbf{u} \times \mathbf{B}+\eta \mathbf{J}+\frac{\delta_{i}}{L_{0}} \frac{\mathbf{J} \times \mathbf{B}}{\rho}+\left(\frac{\delta_{e}}{L_{0}}\right)^{2} \frac{1}{\rho} \cdot\left[\frac{d \mathbf{J}}{d t}+(\mathbf{u} \cdot \nabla) \mathbf{J}\right] .
$$

Here $L_{0}$ is the normalizing length unit, and $\delta_{e}$ and $\delta_{i}$ denote electron and ion inertia respectively; they are related to electron-ion mass ratio by $\left(\frac{\delta_{e}}{\delta_{i}}\right)^{2}=\frac{m_{e}}{m_{i}}$. The term $\mathbf{J} \times \mathbf{B}$ is the so-called Hall term and $\mathbf{J}_{t}+(\mathbf{u} \cdot \nabla) \mathbf{J}$ is the electron inertia term [11].

The equations need to be completed by specifying Ampére's law for the current:

$$
\mathbf{J}=\nabla \times \mathbf{B} .
$$

The MHD equations together with Ohm's law (1.10) and the above Ampére's law are termed as the Hall MHD equations. The Hall MHD equations are non-linear, highorder equations and are extremely complicated to study in a mathematically rigorous manner. There have been various numerical studies of the Hall MHD equations in $[15,19]$ and references therein. However, all these papers tackle the problem from a computational point of view and do not include any rigorous results.

In contrast to the above papers where schemes were designed for the full Hall MHD equations, we adopt a different approach. First, we consider a sub-model: the magnetic induction equations with Hall effect,

$$
\begin{aligned}
\frac{\partial}{\partial t}[\mathbf{B} & \left.+\left(\frac{\delta_{e}}{L_{0}}\right)^{2} \frac{1}{\rho} \nabla \times(\nabla \times \mathbf{B})\right] \\
= & \nabla \times(\mathbf{u} \times \mathbf{B})-\eta \nabla \times(\nabla \times \mathbf{B}) \\
& -\left(\frac{\delta_{e}}{L_{0}}\right)^{2} \frac{1}{\rho} \nabla \times((\mathbf{u} \cdot \nabla)(\nabla \times \mathbf{B})) \\
& -\frac{\delta_{i}}{L_{0}} \frac{1}{\rho} \nabla \times((\nabla \times \mathbf{B}) \times \mathbf{B}) .
\end{aligned}
$$

The magnetic induction equations with Hall effect are augmented with the divergence constraint (1.7) if the initial data is divergence free. Here, the unknowns are the magnetic field $\mathbf{B}$. The velocity field $\mathbf{u}$ is specified a priori. The parameters are as before. Observe that the Magnetic induction equations with Hall effect are still non-linear with the non-linearity being present in the Hall term. Furthermore, the equations contain second-order spatial derivatives (resistivity) and third-order spatial and spatio-temporal derivatives (electron inertia).

In this paper, we investigate the Magnetic induction equations with Hall effect from a mathematical point of view and derive stability estimates. The key tool will be to symmetrize the advection terms in (1.12) using the divergence constraint. See $[3,8,9,13]$ for the use of this technique for the ideal magnetic induction equations and the resistive induction equations. We then derive energy estimates in the Sobolev 
space $H^{1}$ for the Magnetic induction equations with Hall effect with smooth velocity fields.

We also derive stable finite difference schemes for the Magnetic induction equations with Hall effect (1.12) in this paper. These schemes preserve a discrete version of the energy estimate. In particular, we discuss both divergence preserving schemes and schemes that are based on the symmetric form of the equation and may not preserve a discrete version of the divergence constraint. Numerical experiments demonstrating the robust performance of the proposed schemes are presented. To the best of our knowledge, the proposed schemes are the first set of (rigorously proven) stable schemes for the Magnetic induction equations with Hall effect.

The rest of the paper is organized as follows: in Sect. 2, we study the continuous problem for the Magnetic induction equations with Hall effect and show energy estimates. Stable numerical schemes that satisfy a discrete version of the energy inequality are presented in Sect. 3 and we present numerical experiments in Sect. 4.

\section{The continuous problem}

The main aim of this section is to derive energy estimates for the continuous problem corresponding to the Magnetic induction equations with Hall effect (1.12). It turns out that the advection terms in (1.12) are not symmetric and impair the derivation of an energy estimate. We will symmetrize this term (see $[3,8,9]$ ) by using a the vector identity

$$
\nabla \times(\mathbf{u} \times \mathbf{B})=(\mathbf{B} \cdot \nabla) \mathbf{u}-\mathbf{B}(\nabla \cdot \mathbf{u})+\mathbf{u}(\nabla \cdot \mathbf{B})-(\mathbf{u} \cdot \nabla) \mathbf{B} .
$$

We use the divergence constraint (1.7) and subtract $\mathbf{u}(\nabla \cdot \mathbf{B})$ from (1.12) to obtain the symmetric form of the Magnetic induction equations with Hall effect:

$$
\begin{aligned}
\frac{\partial}{\partial t} & {\left[\mathbf{B}+\left(\frac{\delta_{e}}{L_{0}}\right)^{2} \frac{1}{\rho} \nabla \times(\nabla \times \mathbf{B})\right] } \\
= & (\mathbf{B} \cdot \nabla) \mathbf{u}-\mathbf{B}(\nabla \cdot \mathbf{u})-(\mathbf{u} \cdot \nabla) \mathbf{B}-\eta \nabla \times(\nabla \times \mathbf{B}) \\
& -\left(\frac{\delta_{e}}{L_{0}}\right)^{2} \frac{1}{\rho} \nabla \times((\mathbf{u} \cdot \nabla)(\nabla \times \mathbf{B}))-\frac{\delta_{i}}{L_{0}} \frac{1}{\rho} \nabla \times((\nabla \times \mathbf{B}) \times \mathbf{B}) .
\end{aligned}
$$

We show the following energy estimate for the symmetric form of the Magnetic induction equations with Hall effect,

Theorem 2.1 Let the velocity field $\mathbf{u} \in C^{2}\left(\mathbb{R}^{3}\right)$. Furthermore, assume that the solution $\mathbf{B}$ of (2.2) decays to zero at infinity, then following apriori estimate holds:

$$
\begin{aligned}
& \frac{d}{d t}\left(\|\mathbf{B}\|_{L^{2}\left(\mathbb{R}^{3}\right)}^{2}+\left(\frac{\delta_{e}}{L_{0}}\right)^{2} \frac{1}{\rho}\|\nabla \times \mathbf{B}\|_{L^{2}\left(\mathbb{R}^{3}\right)}^{2}\right) \\
& \quad \leq C\left(\|\mathbf{B}\|_{L^{2}\left(\mathbb{R}^{3}\right)}^{2}+\left(\frac{\delta_{e}}{L_{0}}\right)^{2} \frac{1}{\rho}\|\nabla \times \mathbf{B}\|_{L^{2}\left(\mathbb{R}^{3}\right)}^{2}\right)
\end{aligned}
$$

with $C$ being a constant that depend on $\mathbf{u}$ and its derivatives only. 
Proof To simplify the notation and emphasize the symmetry of the equation, we rewrite the term $(\mathbf{B} \cdot \nabla) \mathbf{u}$ in the matrix form obtaining:

$$
\begin{aligned}
\frac{\partial \mathbf{B}}{\partial t}+ & \left(\frac{\delta_{e}}{L_{0}}\right)^{2} \frac{1}{\rho} \nabla \times\left(\nabla \times \frac{\partial \mathbf{B}}{\partial t}\right) \\
= & C \mathbf{B}-(\nabla \cdot \mathbf{u}) \mathbf{B}-(\mathbf{u} \cdot \nabla) \mathbf{B}-\eta \nabla \times(\nabla \times \mathbf{B}) \\
& -\left(\frac{\delta_{e}}{L_{0}}\right)^{2} \frac{1}{\rho} \nabla \times((\mathbf{u} \cdot \nabla)(\nabla \times \mathbf{B}))-\frac{\delta_{i}}{L_{0}} \frac{1}{\rho} \nabla \times((\nabla \times \mathbf{B}) \times \mathbf{B})
\end{aligned}
$$

where

$$
C=\left(\begin{array}{ccc}
\partial_{x} u^{1} & \partial_{y} u^{1} & \partial_{z} u^{1} \\
\partial_{x} u^{2} & \partial_{y} u^{2} & \partial_{z} u^{2} \\
\partial_{x} u^{3} & \partial_{y} u^{3} & \partial_{z} u^{3}
\end{array}\right)
$$

To obtain the $L^{2}$ estimate, we multiply the equation with $\mathbf{B}$ and then we integrate over $\mathbb{R}^{3}$ resulting in

$$
\begin{aligned}
\int_{\mathbb{R}^{3}} & \frac{1}{2} \frac{\partial \mathbf{B}^{2}}{\partial t}+\left(\frac{\delta_{e}}{L_{0}}\right)^{2} \frac{1}{\rho} \mathbf{B} \nabla \times\left(\nabla \times \frac{\partial \mathbf{B}}{\partial t}\right) d x \\
= & \int_{\mathbb{R}^{3}}\left[\mathbf{B}^{\top} C \mathbf{B}-(\nabla \cdot \mathbf{u}) \mathbf{B}^{2}-\frac{1}{2}(\mathbf{u} \cdot \nabla) \mathbf{B}^{2}-\eta \mathbf{B} \nabla \times(\nabla \times \mathbf{B})\right. \\
& \left.-\left(\frac{\delta_{e}}{L_{0}}\right)^{2} \frac{1}{\rho} \mathbf{B} \nabla \times((\mathbf{u} \cdot \nabla)(\nabla \times \mathbf{B}))-\frac{\delta_{i}}{L_{0}} \frac{1}{\rho} \mathbf{B} \nabla \times((\nabla \times \mathbf{B}) \times \mathbf{B})\right] d x .
\end{aligned}
$$

Partial integration yields

$$
\begin{aligned}
\frac{1}{2} \frac{d}{d t}\left(\|\mathbf{B}\|_{L^{2}\left(\mathbb{R}^{3}\right)}^{2}+\left(\frac{\delta_{e}}{L_{0}}\right)^{2} \frac{1}{\rho}\|\nabla \times \mathbf{B}\|_{L^{2}\left(\mathbb{R}^{3}\right)}^{2}\right) \\
=\int_{\mathbb{R}^{3}}\left[\mathbf{B}^{\top} C \mathbf{B}-\frac{1}{2}(\nabla \cdot \mathbf{u}) \mathbf{B}^{2}\right. \\
\quad-\eta(\nabla \times \mathbf{B})^{2}+\frac{1}{2}\left(\frac{\delta_{e}}{L_{0}}\right)^{2} \frac{1}{\rho}(\nabla \cdot \mathbf{u})(\nabla \times \mathbf{B})^{2} \\
\quad-\frac{\delta_{i}}{L_{0}} \frac{1}{\rho} \underbrace{(\nabla \times \mathbf{B})((\nabla \times \mathbf{B}) \times \mathbf{B})}_{=0}] d x \\
=\int_{\mathbb{R}^{3}}\left[\mathbf{B}^{\top} C \mathbf{B}-\frac{1}{2}(\nabla \cdot \mathbf{u}) \mathbf{B}^{2}-\eta(\nabla \times \mathbf{B})^{2}+\frac{1}{2}\left(\frac{\delta_{e}}{L_{0}}\right)^{2} \frac{1}{\rho}(\nabla \cdot \mathbf{u})(\nabla \times \mathbf{B})^{2}\right] d x .
\end{aligned}
$$

We did not consider the boundary terms because they vanish since the solution $B$ is decaying to zero at infinity. Using Cauchy-Schwartz we obtain

$$
\frac{1}{2} \frac{d}{d t}\left(\|\mathbf{B}\|_{L^{2}\left(\mathbb{R}^{3}\right)}^{2}+\left(\frac{\delta_{e}}{L_{0}}\right)^{2}\|\nabla \times \mathbf{B}\|_{L^{2}\left(\mathbb{R}^{3}\right)}^{2}\right)
$$




$$
\leq \frac{1}{2}\left(C_{A}\|\mathbf{B}\|_{L^{2} \mathbb{R}^{3}}^{2}+C_{B}\left(\frac{\delta_{e}}{L_{0}}\right)^{2} \frac{1}{\rho}\|\nabla \times \mathbf{B}\|_{L^{2} \mathbb{R}^{3}}^{2}\right)
$$

where $C_{A} \max _{k, i}\left(\left\|\frac{\partial u_{i}}{\partial x_{k}}\right\|_{L^{\infty}\left(\mathbb{R}^{3}\right)}\right)$ and $C_{B}=\|\nabla \cdot \mathbf{u}\|_{L^{\infty}\left(\mathbb{R}^{3}\right)}$.

We have shown that the Magnetic induction equation with Hall effect in symmetric form possesses an energy estimate. However, the divergence of the solution of (2.2) is not preserved exactly. Nevertheless, we have the following estimate:

Theorem 2.2 Let $\mathbf{u} \in C^{2}\left(\mathbb{R}^{3}\right)$ Furthermore, assume that the divergence of the solution of (2.2), $\nabla \cdot \mathbf{B}$, decays to zero at infinity, then the following apriori estimate holds:

$$
\frac{d}{d t}\|\nabla \cdot \mathbf{B}\|_{L^{2}\left(\mathbb{R}^{3}\right)} \leq C\|\nabla \cdot \mathbf{B}\|_{L^{2}\left(\mathbb{R}^{3}\right)}
$$

with $C$ being a constant that depend on $\mathbf{u}$ and its derivatives only.

Proof Applying the divergence operator on (2.2) we obtain

$$
\frac{\partial \nabla \cdot \mathbf{B}}{\partial t}=-\nabla \cdot(\mathbf{u}(\nabla \cdot \mathbf{B}))
$$

using vector identity we can rewrite the right hand side of the equation

$$
\frac{\partial \nabla \cdot \mathbf{B}}{\partial t}=-\mathbf{u} \cdot \nabla(\nabla \cdot \mathbf{B})-(\nabla \cdot \mathbf{B})(\nabla \cdot \mathbf{u})
$$

Multiplying this equation by $\nabla \cdot \mathbf{B}$ and integrating it over $\mathbb{R}^{3}$ we obtain

$$
\frac{1}{2} \frac{d}{d t}\|\nabla \cdot \mathbf{B}\|_{L^{2}\left(\mathbb{R}^{3}\right)}=-\frac{1}{2} \int_{\mathbb{R}^{3}} \mathbf{u} \cdot\left(\nabla(\nabla \cdot \mathbf{B})^{2}\right) d x-\int_{\mathbb{R}^{3}}(\nabla \cdot \mathbf{u})(\nabla \cdot \mathbf{B})^{2} d x,
$$

with partial integration we obtain

$$
\begin{aligned}
\frac{1}{2} \frac{d}{d t}\|\nabla \cdot \mathbf{B}\|_{L^{2}\left(\mathbb{R}^{3}\right)} & =\frac{1}{2} \int_{\mathbb{R}^{3}}(\nabla \cdot \mathbf{u})(\nabla \cdot \mathbf{B})^{2} d x-\frac{1}{2} \int_{\mathbb{R}^{3}}(\mathbf{u} \cdot \mathbf{n})(\nabla \cdot \mathbf{B})^{2} d s \\
& \left.\leq \frac{1}{2}\|\nabla \cdot \mathbf{u}\|_{L^{\infty}\left(\mathbb{R}^{3}\right)} \| \nabla \cdot \mathbf{B}\right) \|_{L^{2}\left(\mathbb{R}^{3}\right)}^{2} .
\end{aligned}
$$

Corollary 2.1 If the conditions for Theorems 2.1 and 2.2 hold and the initial data $\mathbf{B} \in H^{1}\left(\mathbb{R}^{3}\right)$, then the estimates imply that $\mathbf{B} \in L^{\infty}\left((0, T), H^{1}\left(\mathbb{R}^{3}\right)\right)$.

Remark 2.1 The divergence transport equation (2.6) implies that the divergence remains zero if the initial data has zero divergence. If we assume that the initial data is divergence free, the solutions of the symmetric form (2.2) have zero divergence are also weak solutions of the non-symmetric form (1.12) of the Magnetic induction equations with Hall effect. 


\section{Numerical schemes}

In this section, we design numerical schemes that satisfy a discrete version of the energy estimates of the last section. The computational domain is $\Omega=\left[0, L_{x}\right] \times$ $\left[0, L_{y}\right] \times\left[0, L_{z}\right]$ and we define a uniform mesh of $N_{x}$ times $N_{y}$ times $N_{z}$ points with coordinates $x_{i}=i \Delta x, y_{j}=j \Delta y$ and $z_{k}=k \Delta z$. In this case $\Delta x=L_{x} /\left(N_{x}-1\right)$, $\Delta y=L_{y} /\left(N_{y}-1\right)$ and $\Delta z=L_{z} /\left(N_{z}-1\right)$ are mesh widths.

The point values of the magnetic and velocity fields are

$$
\tilde{\mathbf{B}}_{i, j, k} \sim \mathbf{B}\left(x_{i}, y_{j}, z_{k}\right), \quad \tilde{\mathbf{u}}_{i, j, k} \sim \mathbf{u}\left(x_{i}, y_{j}, z_{k}\right) .
$$

We will provide a very general discrete formulation and specify the necessary requirement that the discrete derivative should posses. As in $[8,9,13]$, the key requirement for the discrete derivative is to satisfy a summation by parts (SBP) condition. The exact form of operators satisfying the requirements are presented in Appendix.

We start with one dimensional discrete derivatives using grid functions in vector form, i.e., $w=\left(w_{0}, \ldots w_{N_{x}-1}\right)^{\top}$. An approximation of the $x$ spatial derivative, $D_{x}$ possesses the summation by parts property (see $[2,10,12,16,17])$ if it can be written as $D_{x}=P_{x}^{-1} Q_{x}$, where $P_{x}$ is a diagonal $N_{x} \times N_{x}$ positive definite matrix and $Q_{x}$ an $N_{x} \times N_{x}$ matrix satisfying:

$$
Q_{x}+Q_{x}^{\top}=R_{N_{x}}-L_{N_{x}}
$$

where $R_{N_{x}}$ and $L_{N_{x}}$ are $N_{x} \times N_{x}$ matrices: $\operatorname{diag}(0, \ldots, 0,1)$ and $\operatorname{diag}(1,0, \ldots, 0)$.

The operator $P$ defines an inner product

$$
(v, w)_{P_{x}}=v^{\top} P_{x} w
$$

with the associated norm $\|w\|_{P}=(w, w)_{P_{x}}^{1 / 2}$ that is equivalent to the norm $\|w\|=$ $\left(\Delta x \sum_{k} w_{k}^{2}\right)^{1 / 2}$.

Next, we define averaging operators such that we can obtain an approximate form of the chain rule.

We define symmetric averaging operators as

$$
\left(A_{x} w\right)_{i}=\sum_{k=-q}^{q} \alpha_{k} w_{i+k}
$$

with $\sum_{k=-q}^{q} \alpha_{k}=1$ and $\alpha_{-i}=\alpha_{i}$.

In [13], the following discrete chain rule was shown,

Lemma 3.1 Given any smooth function $u(x)$, we denote its restriction on the grid as $\bar{u}$ and let be $w$ a grid function. Then we can define an averaging operator $\bar{A}_{x}$ coupled to $D_{x}$ such that

$$
D_{x}(\bar{u} \circ w)=\bar{u} \circ D_{x}(w)+D_{x}(\bar{u}) \circ \bar{A}_{x}(w)+\tilde{w},
$$

where $(u \circ w)_{i, j, k}=u_{i, j, k} w_{i, j, k}, \bar{A}_{x}(w)=\sum_{k=-q}^{q} k \beta_{k} w_{i+k}$ and $\|\tilde{w}\|_{P} \leq C \Delta x\|w\|_{P}$. 
Proof The discrete differential operator acting on a grid function $w_{i}$ can also be written through sums:

$$
\left(D_{x} w\right)_{i}=\frac{1}{\Delta x} \sum_{k=-q}^{q} \beta_{k} w_{i+k},
$$

with $\sum_{k=-q}^{q} \beta_{k}=0$ and $\sum_{k=-q}^{q} k \beta_{k}=1$. Then the residual $\tilde{w}$ is given by

$$
\begin{aligned}
\tilde{w}_{i} & =D_{x}(\bar{u} \circ w)_{i}-\left(\bar{u} \circ D_{x} w\right)_{i}-\left(D_{x}(\bar{u}) \circ \bar{A}_{x}(w)\right)_{i} \\
& =\frac{1}{\Delta x} \sum_{k=-q}^{q} \beta_{k} \bar{u}_{i+k} w_{i+k}-\frac{\bar{u}_{i}}{\Delta x} \sum_{k=-q}^{q} \beta_{k} w_{i+k}-\frac{1}{\Delta x}\left(\sum_{l=-q}^{q} \beta_{l} \bar{u}_{i+l}\right) \sum_{k=-q}^{q} k \beta_{k} w_{i+k} .
\end{aligned}
$$

We expand $\bar{u}$ with Taylor-expansion

$$
\bar{u}_{i+k}=\bar{u}_{i}+\Delta x k \bar{u}_{i}^{\prime}+\frac{1}{2} \Delta x^{2} k^{2} c_{k}^{i}
$$

where $\mathfrak{c}_{k}^{i}=\bar{u}_{i}^{\prime \prime}\left(\xi_{k}\right)$ obtaining

$$
\begin{aligned}
\tilde{w}_{i}= & \frac{\bar{u}_{i}}{\Delta x} \bar{u}_{i} \sum_{k=-q}^{q} \beta_{k} w_{i+k}+\bar{u}_{i}^{\prime} \sum_{k=-q}^{q} k \beta_{k} w_{i+k}+\Delta x \sum_{k=-q}^{q} k^{2} \beta_{k} c_{k}^{i} w_{i+k} \\
& -\frac{\bar{u}_{i}}{\Delta x} \bar{u}_{i} \sum_{k=-q}^{q} \beta_{k} w_{i+k} \\
& -\left(\bar{u}_{i}^{\prime}+\Delta x \sum_{l=-q}^{q} l^{2} \beta_{l} c_{l}^{i}\right) \sum_{k=-q}^{q} k \beta_{k} w_{i+k} \\
= & \Delta x \sum_{k=-q}^{q} \gamma_{k}^{i} w_{i+k} .
\end{aligned}
$$

Here $\gamma_{k}^{i}=k \beta_{k} \frac{k c_{k}-\sum_{l=-q}^{q} l^{2} \beta_{l} c_{l}^{i}}{2}$. Since the $\gamma_{k}^{i}$ are bounded, we have that

$$
\|\tilde{w}\|_{P_{x}} \leq \Delta x C\|w\|_{P_{x}},
$$

where $C$ depends only on the maximum of $\gamma_{k}^{i}$ and on the norm $P_{x}$.

We show the following lemma claiming that differential operators commute with averaging operators,

Lemma 3.2 The discrete differential operator $D_{x}$ and the averaging operator $A_{x}$ commute. 
Proof We write the discrete differential operator acting on a grid function $w_{i}$ through sums:

$$
\left(D_{x} w\right)_{i}=\frac{1}{\Delta x} \sum_{k^{\prime}=-q^{\prime}}^{q^{\prime}} \beta_{k^{\prime}} \cdot w_{i+k^{\prime}} .
$$

Then applying the two operators consecutively we obtain

$$
\begin{aligned}
\left(D_{x}\left(A_{x} w\right)\right)_{i} & =\frac{1}{\Delta x} \sum_{k^{\prime}=-q^{\prime}}^{q^{\prime}} \beta_{k^{\prime}}\left(A_{x} w\right)_{i+k^{\prime}}=\frac{1}{\Delta x} \sum_{k^{\prime}=-q^{\prime}}^{q^{\prime}} \beta_{k^{\prime}} \sum_{k=-q}^{q} \alpha_{k} w_{i+k^{\prime}+k} \\
& =\frac{1}{\Delta x} \sum_{k^{\prime}=-q^{\prime}}^{q^{\prime}} \sum_{k=-q}^{q} \beta_{k^{\prime}} \alpha_{k} w_{i+k^{\prime}+k}=\frac{1}{\Delta x} \sum_{k=-q}^{q} \sum_{k^{\prime}=-q^{\prime}}^{q^{\prime}} \beta_{k^{\prime}} \alpha_{k} w_{i+k^{\prime}+k} \\
& =\frac{1}{\Delta x} \sum_{k=-q}^{q} \alpha_{k} \sum_{k^{\prime}=-q^{\prime}}^{q^{\prime}} \beta_{k^{\prime}} w_{i+k^{\prime}+k} \\
& =\sum_{k=-q}^{q} \alpha_{k}\left(D_{x} w\right)_{i+k}=\left(A_{x}\left(D_{x} w\right)\right)_{i} .
\end{aligned}
$$

We use the above one dimensional operators to build the multidimensional operators i.e., mappings of three dimensional grid functions $w\left(x_{i}, y_{j}, z_{k}\right)=w_{i, j . k}$ to a column vector

$$
w=\left(w_{0,0,0}, w_{0,0,1}, \ldots, w_{0,0, N_{z}}, w_{0,1,0}, \ldots, w_{N_{x}, N_{y}, N_{z}}\right)^{\top} .
$$

We define the discrete differential operators and averages

$$
\begin{aligned}
& \mathfrak{d}_{x}=D_{x} \otimes A_{y} \otimes A_{z}, \\
& \mathfrak{d}_{y}=A_{x} \otimes D_{y} \otimes A_{z}, \\
& \mathfrak{d}_{z}=A_{x} \otimes A_{y} \otimes D_{z}, \\
& \mathscr{A}_{x}=\bar{A}_{x} \otimes A_{y} \otimes A_{z}, \\
& \mathscr{A}_{y}=A_{x} \otimes \bar{A}_{y} \otimes A_{z}, \\
& \mathscr{A}_{z}=A_{x} \otimes A_{y} \otimes \bar{A}_{z} .
\end{aligned}
$$

Here $\otimes$ is the Kronecker product. We also extend the inner product by $\mathbf{P}=\mathbf{P}_{x} \otimes \mathbf{P}_{y} \otimes$ $\mathbf{P}_{z}$ with the corresponding norm $\|w\|_{\mathbf{P}}=(w, w)_{\mathbf{P}}^{1 / 2}$.

We generalize the one dimensional operators to three dimensions using the averaging operators $A_{x}, A_{y}$ and $A_{z}$. Setting the averaging operators to the identity mapping allows us to recover the standard version of one dimensional discrete operators. However, we will use a more general form of the averaging operator that will allow us to include a larger group of difference operators including the divergence preserving operators of [18] and some of the divergence preserving operators proposed in [14]. 
We can expand Lemma 3.1 in three dimensions:

Corollary 3.1 Given any smooth function $\bar{u}(x)$ as above, then

$$
\left\|\mathfrak{d}_{x}(u \circ w)-u \circ \mathfrak{d}_{x} w\right\|_{\mathbf{P}} \leq C\|w\|_{\mathbf{P}}
$$

where $C$ a constant which depends on the first order derivative of $u$.

Proof The proof of this corollary is similar to the one done before only using a substitution with a Taylor expansion of degree one.

The summation by parts property of the differential operators, coupled with the inner product $\mathbf{P}$ will result in a discrete version on integration by parts:

Lemma 3.3 For any grids function $v$ and $w$, we have

$$
\begin{aligned}
& \left(v, \mathfrak{d}_{x} w\right)_{\mathbf{P}}+\left(\mathfrak{d}_{x} v, w\right)_{\mathbf{P}}=v^{\top}(\mathscr{R}-\mathscr{L}) w, \\
& \left(v, \mathfrak{d}_{y} w\right)_{\mathbf{P}}+\left(\mathfrak{d}_{y} v, w\right)_{\mathbf{P}}=v^{\top}(\mathscr{U}-\mathscr{D}) w, \\
& \left(v, \mathfrak{d}_{z} w\right)_{\mathbf{P}}+\left(\mathfrak{d}_{z} v, w\right)_{\mathbf{P}}=v^{\top}(\mathscr{T}-\mathscr{B}) w,
\end{aligned}
$$

where $\mathscr{R}=R_{N_{x}} \otimes P_{y} A_{y} \otimes P_{z} A_{z}, \mathscr{L}=L_{N_{x}} \otimes P_{y} A_{y} \otimes P_{z} A_{z}, \mathscr{U}=P_{x} A_{x} \otimes R_{N_{y}} \otimes$ $P_{z} A_{z}, \mathscr{D}=P_{x} A_{y} \otimes L_{R_{y}} \otimes P_{z} A_{z}, \mathscr{T}=P_{x} A_{y} \otimes P_{y} A_{y} \otimes R_{N_{z}}$ and $\mathscr{B}=P_{x} A_{y} \otimes$ $P_{y} A_{y} \otimes L_{N_{z}}$.

Proof Since $A_{k}$ 's are symmetric and $P_{k}$ 's diagonal, we can calculate

$$
\begin{aligned}
\left(v, \mathfrak{d}_{x} w\right)_{\mathbf{P}}+\left(\mathfrak{d}_{x} v, w\right)_{\mathbf{P}} \\
=v^{\top}\left(P_{x} \otimes P_{y} \otimes P_{z}\right)\left(P_{x}^{-1} Q_{x} \otimes A_{y} \otimes A_{z}\right) w \\
\quad+\left(\left(P_{x}^{-1} Q_{x} \otimes A_{y} \otimes A_{z}\right) v\right)^{\top}\left(P_{x} \otimes P_{y} \otimes P_{z}\right) w \\
=v^{\top}\left(Q_{x} \otimes A_{y}^{\top} P_{y} \otimes A_{z}^{\top} P_{z}\right) w+v^{\top}\left(Q_{x}^{\top} \otimes P_{y} A_{y} \otimes P_{z} A_{z}\right) w \\
=v^{\top}\left(\left(Q_{x}^{\top}+Q_{x}\right) \otimes P_{y} A_{y} \otimes P_{z} A_{z}\right) w \\
=v^{\top}\left(R_{N_{x}} \otimes P_{y} A_{y} \otimes P_{z} A_{z}\right) w-v^{\top}\left(L_{N_{x}} \otimes P_{y} A_{y} \otimes P_{z} A_{z}\right) w .
\end{aligned}
$$

The proof for the other space directions follow analogously.

The right hand side of the above equation represents the evaluation of the grid function on the boundary of $\Omega$.

Until now all our analysis was for scalar operators, we extend it to vector-valued discrete differential operators below. 
Corollary 3.2 We derive from the scalar summation by parts rules for scalar fields

$$
\begin{aligned}
(\tilde{\mathbf{v}}, \mathbf{D} \cdot \tilde{\mathbf{w}})_{\mathbf{P}} & =-(\mathbf{D} \tilde{\mathbf{v}}, \tilde{\mathbf{w}})_{\mathbf{P}}+\sum_{i} \hat{v}_{i} \mathscr{S}_{i} \hat{w}_{i}, \\
(\tilde{\mathbf{v}}, \mathbf{D} \times \tilde{\mathbf{w}})_{\mathbf{P}} & =(\mathbf{D} \times \tilde{\mathbf{v}}, \tilde{\mathbf{w}})_{\mathbf{P}}+\sum_{i, j, k} \varepsilon_{i, j, k} \hat{v}_{i} \mathscr{S}_{j} \hat{w}_{k},
\end{aligned}
$$

where $\mathbf{D}=\left(\mathfrak{d}_{x}, \mathfrak{d}_{y}, \mathfrak{d}_{z}\right)^{\top}, \mathscr{S}_{1}=\mathscr{L}-\mathscr{R}, \mathscr{S}_{2}=\mathscr{U}-\mathscr{D}, \mathscr{S}_{3}=\mathscr{T}-\mathscr{B}$ and $\varepsilon_{i, j, k}$ is the Levi-Civita symbol.

Proof The proof of this corollary is given by the direct application of the previous theorem on discrete vector fields.

In the continuous case, the key property for the preservation of divergence is the identity:

$$
\nabla \cdot(\nabla \times \mathbf{w})=0
$$

for all $\mathbf{w} \in\left(C^{2}\left(\mathbb{R}^{3}\right)\right)^{3}$. From Lemma 3.2 and the definition of Kronecker product, one observes that the difference operators $\mathfrak{d}_{x}, \mathfrak{d}_{y}$ and $\mathfrak{d}_{z}$ commute, we can show that is also the case for the discrete differential operator:

Corollary 3.3 Every grid function $\hat{\mathbf{w}}_{i, j, k}$ coupled with $\mathbf{D}=\left(\mathfrak{d}_{x}, \mathfrak{d}_{y}, \mathfrak{d}_{z}\right)^{\top}$ satisfies

$$
\mathbf{D} \cdot(\mathbf{D} \times \hat{\mathbf{w}})=0 .
$$

The proof of this corollary is straightforward.

Generalizing Lemma 3.3 presented in [8] for the vector operator $\mathbf{D}$ :

\section{Lemma 3.4 If $\tilde{\mathbf{u}}$ is a vector grid function}

$$
(\mathbf{v},(\tilde{\mathbf{u}} \cdot \mathbf{D}) \circ \tilde{\mathbf{v}})_{\mathbf{P}}=\frac{1}{2}\left(\tilde{\mathbf{v}},(\tilde{\mathbf{u}} \mathbf{D}) \circ \tilde{\mathbf{v}}-\sum_{i} \mathbf{D}\left(\left(\tilde{\mathbf{u}}^{i}\right) \circ \tilde{\mathbf{v}}\right)\right)_{\mathbf{P}}+\frac{1}{2} \sum_{i} \tilde{\mathbf{v}}^{\top} \mathscr{S}_{i}\left(\left(\tilde{\mathbf{u}}^{i}\right) \circ \tilde{\mathbf{v}}\right)
$$

Proof See the proof in [9] and apply it on each component of $\tilde{\mathbf{u}} \cdot \tilde{\mathbf{D}}$.

Now we have all the ingredients to present two different classes of numerical schemes. The first set of schemes termed as symmetric schemes will discretize the symmetric version of the Hall magnetic induction equation (2.2). The second set of schemes will discrete the non-symmetric version (1.12) of the Magnetic induction equations with Hall effect and will preserve a discrete version of divergence. Hence, we term it divergence preserving scheme. 


\subsection{Symmetric scheme}

We discretize the symmetric form (2.2) of the Magnetic induction equations with Hall effect and define a semi-discrete numerical scheme as

$$
\begin{aligned}
\frac{d}{d t}\left(\tilde{\mathbf{B}}_{i, j, k}+\left(\frac{\delta_{e}}{L_{0}}\right)^{2} \frac{1}{\rho}(\mathbf{D} \times \mathbf{D} \times \tilde{\mathbf{B}})_{i, j, k}\right) \\
=\mathscr{A} \mathscr{V}(\tilde{\mathbf{B}}, \tilde{\mathbf{u}})-\left(\tilde{\mathbf{u}}_{i, j, k} \cdot \mathbf{D}\right) \tilde{\mathbf{B}}_{i, j, k} \\
\quad-\eta\left(\mathbf{D} \times\left(\mathbf{D} \times \tilde{\mathbf{B}}_{i, j, k}\right)\right)-\left(\frac{\delta_{e}}{L_{0}}\right)^{2} \frac{1}{\rho} \mathbf{D} \times\left(\left(\tilde{\mathbf{u}}_{i, j, k} \cdot \mathbf{D}\right)\left(\mathbf{D} \times \tilde{\mathbf{B}}_{i, j, k}\right)\right) \\
\quad-\frac{\delta_{i}}{L_{0}} \frac{1}{\rho} \mathbf{D} \times\left(\left(\mathbf{D} \times \tilde{\mathbf{B}}_{i, j, k}\right) \times \tilde{\mathbf{B}}_{i, j, k}\right)
\end{aligned}
$$

where

$$
\begin{aligned}
& \mathscr{A} \mathscr{V}(\tilde{\mathbf{B}}, \tilde{\mathbf{u}})=\left(\overline{\mathscr{A}}\left(\tilde{\mathbf{B}}_{i, j, k}\right) \cdot \mathbf{D}\right) \tilde{\mathbf{u}}_{i, j, k}-\overline{\mathscr{A}}_{x}\left(\tilde{\mathbf{B}}_{i, j, k}\right) \mathfrak{d}_{x}\left(\tilde{\mathbf{u}}_{i, j, k}^{1}\right)-\overline{\mathscr{A}}_{y}\left(\tilde{\mathbf{B}}_{i, j, k}\right) \mathfrak{d}_{y}\left(\tilde{\mathbf{u}}_{i, j, k}^{2}\right) \\
&-\overline{\mathscr{A}}_{z}\left(\tilde{\mathbf{B}}_{i, j, k}\right) \mathfrak{d}_{z}\left(\tilde{\mathbf{u}}_{i, j, k}^{3}\right), \\
& \overline{\mathscr{A}}\left(\tilde{\mathbf{B}}_{i, j, k}\right)=\left(\overline{\mathscr{A}}_{x}\left(\tilde{\mathbf{B}}_{i, j, k}^{1}\right), \overline{\mathscr{A}}_{y}\left(\tilde{\mathbf{B}}_{i, j, k}^{2}\right), \overline{\mathscr{A}}_{z}\left(\tilde{\mathbf{B}}_{i, j, k}^{3}\right)\right)^{\top} .
\end{aligned}
$$

The term $\mathscr{A} \mathscr{V}$ represent the discretisation of $(\mathbf{B} \cdot \nabla) \mathbf{u}-\mathbf{B}(\nabla \cdot \mathbf{u})$. We estimate it below,

Lemma 3.5 For $\tilde{\mathbf{B}}_{i, j, k}$ grid function and $\tilde{\mathbf{u}}_{i, j, k}$ a bounded grid function, we have

$$
(\tilde{\mathbf{B}}, \mathscr{A} \mathscr{V}(\tilde{\mathbf{B}}, \tilde{\mathbf{u}}))_{\mathbf{P}} \leq C\|\tilde{\mathbf{B}}\|_{\mathbf{P}}^{2},
$$

where $C$ depends on $\tilde{\mathbf{u}}$ and its discrete derivative only.

Proof We write (3.15) component-wise and using the triangle inequality, we obtain

$$
\begin{aligned}
(\tilde{\mathbf{B}}, \mathscr{A} \mathscr{V}(\tilde{\mathbf{B}}, \tilde{\mathbf{u}}))_{\mathbf{P}} \leq & \sum_{i=1}^{3}\left|\left(\mathbf{B}^{\mathbf{i}}, \overline{\mathscr{A}}_{x}\left(\tilde{\mathbf{B}}^{1}\right) \mathfrak{d}_{x} \tilde{\mathbf{u}}^{i}\right)_{\mathbf{P}}\right|+\left|\left(\mathbf{B}^{\mathbf{i}}, \overline{\mathscr{A}}_{y}\left(\tilde{\mathbf{B}}^{2}\right) \mathfrak{d}_{y} \tilde{\mathbf{u}}^{i}\right)_{\mathbf{P}}\right| \\
& +\left|\left(\mathbf{B}^{\mathbf{i}}, \overline{\mathscr{A}}_{z}\left(\tilde{\mathbf{B}}^{3}\right) \mathfrak{d}_{z} \tilde{\mathbf{u}}^{i}\right)_{\mathbf{P}}\right|+\left|\left(\mathbf{B}^{\mathbf{i}}, \overline{\mathscr{A}}_{x}\left(\tilde{\mathbf{B}}^{i}\right) \mathfrak{d}_{x} \tilde{\mathbf{u}}^{1}\right)_{\mathbf{P}}\right| \\
& +\left|\left(\mathbf{B}^{\mathbf{i}}, \overline{\mathscr{A}}_{y}\left(\tilde{\mathbf{B}}^{i}\right) \mathfrak{d}_{y} \tilde{\mathbf{u}}^{2}\right)_{\mathbf{P}}\right|+\left|\left(\tilde{\mathbf{B}}^{\mathbf{i}}, \overline{\mathscr{A}}_{z}\left(\tilde{\mathbf{B}}^{i}\right) \mathfrak{d}_{z} \tilde{\mathbf{u}}^{3}\right)_{\mathbf{P}}\right|
\end{aligned}
$$

Since the discrete derivative of $\tilde{\mathbf{u}}$ are bounded, we can extract its maximum from the $\mathbf{P}$ norms and obtain

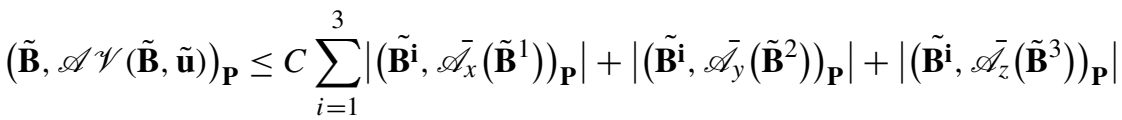

$$
\begin{aligned}
& +\left|\left(\tilde{\mathbf{B}^{\mathbf{i}}}, \overline{\mathscr{A}}_{x}\left(\tilde{\mathbf{B}}^{i}\right)\right)_{\mathbf{P}}\right|+\left|\left(\tilde{\mathbf{B}^{\mathbf{i}}}, \overline{\mathscr{A}}_{y}\left(\tilde{\mathbf{B}}^{i}\right)\right)_{\mathbf{P}}\right|+\left|\left(\tilde{\mathbf{B}}^{\mathbf{i}}, \overline{\mathscr{A}}_{z}\left(\tilde{\mathbf{B}}^{i}\right)\right)_{\mathbf{P}}\right|
\end{aligned}
$$




$$
\begin{aligned}
& \leq C\left(2\left(\left|\left(\tilde{\mathbf{B}} \mathbf{1}, \overline{\mathscr{A}}_{x}\left(\tilde{\mathbf{B}}^{1}\right)\right)_{\mathbf{P}}\right|+\left|\left(\tilde{\mathbf{B}^{2}}, \overline{\mathscr{A}}_{y}\left(\tilde{\mathbf{B}}^{2}\right)\right)_{\mathbf{P}}\right|+\left|\left(\tilde{\mathbf{B}^{3}}, \overline{\mathscr{A}}_{z}\left(\tilde{\mathbf{B}}^{3}\right)\right)_{\mathbf{P}}\right|\right)\right. \\
& +\left|\left(\tilde{\mathbf{B}^{2}}, \overline{\mathscr{A}}_{x}\left(\tilde{\mathbf{B}}^{1}\right)\right)_{\mathbf{P}}\right|+\left|\left(\tilde{\mathbf{B}^{3}}, \overline{\mathscr{A}}_{x}\left(\tilde{\mathbf{B}}^{1}\right)\right)_{\mathbf{P}}\right|+\left|\left(\tilde{\mathbf{B}^{\mathbf{1}}}, \overline{\mathscr{A}}_{y}\left(\tilde{\mathbf{B}}^{2}\right)\right)_{\mathbf{P}}\right| \\
& \left.+\left|\left(\tilde{\mathbf{B}^{\mathbf{3}}}, \overline{\mathscr{A}}_{y}\left(\tilde{\mathbf{B}}^{2}\right)\right)_{\mathbf{P}}\right|+\left|\left(\tilde{\mathbf{B}^{\mathbf{1}}}, \overline{\mathscr{A}}_{z}\left(\tilde{\mathbf{B}}^{3}\right)\right)_{\mathbf{P}}\right|+\left|\left(\tilde{\mathbf{B}^{2}}, \overline{\mathscr{A}}_{z}\left(\tilde{\mathbf{B}}^{3}\right)\right)_{\mathbf{P}}\right|\right) \text {. }
\end{aligned}
$$

Take the first term and first use the equivalence of inner product, then write the averaging operator through summation, and finally using the Cauchy inequality yields

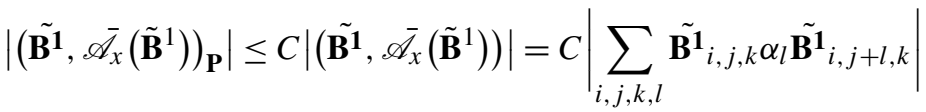

$$
\begin{aligned}
& \leq C \frac{\max _{l}\left(\alpha_{l}\right)}{2} \sum_{i, j, k, l}\left[\left(\tilde{\mathbf{B}}_{i, j, k}\right)^{2}+\left(\tilde{\mathbf{B}}_{i, j+l, k}\right)^{2}\right] \leq C\|\tilde{\mathbf{B}}\|^{2} .
\end{aligned}
$$

Repeating this procedure for all the term of the sum, yields to

$$
(\tilde{\mathbf{B}}, \mathscr{A} \mathscr{V}(\tilde{\mathbf{B}}, \tilde{\mathbf{u}}))_{\mathbf{P}} \leq C\|\tilde{\mathbf{B}}\|^{2}
$$

The use of the equivalence between Euclidean and $\mathbf{P}$ norms conclude the proof of lemma.

This lemma will allow us to is now prove the energy stability of the scheme. The main theorem is:

Theorem 3.1 Let $\tilde{\mathbf{u}}_{i, j, k}=\mathbf{u}\left(x_{i}, y_{j}, z_{k}\right)$ be the point evaluation of a function $u \in C^{2}$ and let the approximate solutions $\tilde{\mathbf{B}}$ of (3.11) go to zero at infinity, then the following estimates hold

$$
\frac{d}{d t}\left(\|\tilde{\mathbf{B}}\|_{\mathbf{P}}^{2}+\left(\frac{\delta_{e}}{L_{0}}\right)^{2} \frac{1}{\rho}\|\mathbf{D} \times \tilde{\mathbf{B}}\|_{\mathbf{P}}^{2}\right) \leq C\left(\|\tilde{\mathbf{B}}\|_{\mathbf{P}}^{2}+\left(\frac{\delta_{e}}{L_{0}}\right)^{2} \frac{1}{\rho}\|\mathbf{D} \times \tilde{\mathbf{B}}\|_{\mathbf{P}}^{2}\right)
$$

with $C$ a constant that depend on $\mathbf{u}$ and its derivative only.

Proof Multiplying both sides of the scheme (3.11) by $\tilde{\mathbf{B}}$ yields

$$
\begin{aligned}
\frac{1}{2} \frac{d}{d t}\|\tilde{\mathbf{B}}\|_{\mathbf{P}}^{2}+\left(\frac{\delta_{e}}{L_{0}}\right)^{2} \frac{1}{\rho}\left(\tilde{\mathbf{B}},\left(\mathbf{D} \times \mathbf{D} \times \frac{d}{d t} \tilde{\mathbf{B}}\right)\right)_{\mathbf{P}} \\
=(\tilde{\mathbf{B}}, \mathscr{A} \mathscr{V}(\tilde{\mathbf{B}}, \tilde{\mathbf{u}}))_{\mathbf{P}}-(\tilde{\mathbf{B}},(\tilde{\mathbf{u}} \cdot \mathbf{D}) \circ \tilde{\mathbf{B}})_{\mathbf{P}} \\
\quad-\eta(\tilde{\mathbf{B}}, \mathbf{D} \times(\mathbf{D} \times \tilde{\mathbf{B}}))_{\mathbf{P}}-\left(\frac{\delta_{e}}{L_{0}}\right)^{2} \frac{1}{\rho}\left(\tilde{\mathbf{B}}, \mathbf{D} \times((\tilde{\mathbf{u}} \cdot \mathbf{D}) \circ(\mathbf{D} \times \tilde{\mathbf{B}}))_{\mathbf{P}}\right) \\
\quad-\frac{\delta_{i}}{L_{0}} \frac{1}{\delta}(\tilde{\mathbf{B}}, \mathbf{D} \times((\mathbf{D} \times \tilde{\mathbf{B}}) \times \tilde{\mathbf{B}}))_{\mathbf{P}} .
\end{aligned}
$$

Using summation by parts of Corollary 3.2 and Lemma 3.4 


$$
\begin{aligned}
\frac{d}{d t} \frac{1}{2}( & \left.\|\tilde{\mathbf{B}}\|_{\mathbf{P}}^{2}+\left(\frac{\delta_{e}}{L_{0}}\right)^{2} \frac{1}{\rho}\|\mathbf{D} \times \tilde{\mathbf{B}}\|_{\mathbf{P}}^{2}\right) \\
= & (\tilde{\mathbf{B}}, \mathscr{A} \mathscr{V}(\tilde{\mathbf{B}}, \tilde{\mathbf{u}}))_{\mathbf{P}}-\frac{1}{2}(\tilde{\mathbf{B}},(\tilde{\mathbf{u}} \cdot \mathbf{D}) \circ \tilde{\mathbf{B}} \\
& \left.-\sum_{i} \mathbf{D}\left(\tilde{\mathbf{u}}^{i} \circ \tilde{\mathbf{B}}\right)\right)_{\mathbf{P}}-\eta\|\mathbf{D} \times \tilde{\mathbf{B}}\|_{\mathbf{P}}^{2}-\left(\frac{\delta_{e}}{L_{0}}\right)^{2} \frac{1}{\rho}(\mathbf{D} \times \tilde{\mathbf{B}},(\tilde{\mathbf{u}} \cdot \mathbf{D}) \circ(\mathbf{D} \times \tilde{\mathbf{B}}))_{\mathbf{P}} \\
& -\frac{\delta_{i}}{L_{0}} \frac{1}{\delta} \underbrace{(\mathbf{D} \times \tilde{\mathbf{B}},(\mathbf{D} \times \tilde{\mathbf{B}}) \times \tilde{\mathbf{B}})_{\mathbf{P}}}_{=0}=(\tilde{\mathbf{B}}, \mathscr{A} \mathscr{V}(\tilde{\mathbf{B}}, \tilde{\mathbf{u}}))_{\mathbf{P}}-\frac{1}{2}(\tilde{\mathbf{B}},(\tilde{\mathbf{u}} \cdot \mathbf{D}) \circ \tilde{\mathbf{B}} \\
& \left.-\sum_{i} \mathbf{D}\left(\tilde{\mathbf{u}}^{i} \circ \tilde{\mathbf{B}}\right)\right)_{\mathbf{P}}-\eta\|\mathbf{D} \times \tilde{\mathbf{B}}\|_{\mathbf{P}}^{2}-\left(\frac{\delta_{e}}{L_{0}}\right)^{2} \frac{1}{\rho}(\mathbf{D} \times \tilde{\mathbf{B}},(\tilde{\mathbf{u}} \cdot \mathbf{D}) \circ(\mathbf{D} \times \tilde{\mathbf{B}}) \\
& \left.-\sum_{i} \mathbf{D}\left(\tilde{\mathbf{u}}^{i} \circ(\mathbf{D} \times \tilde{\mathbf{B}})\right)\right)_{\mathbf{P}} .
\end{aligned}
$$

All the boundary terms have been neglected since the data decay to 0 at infinity.

Using Lemma 3.4 and Lemma 3.5 we obtain

$$
\frac{d}{d t} \frac{1}{2}\left(\|\tilde{\mathbf{B}}\|_{\mathbf{P}}^{2}+\left(\frac{\delta_{e}}{L_{0}}\right)^{2} \frac{1}{\rho}\|\mathbf{D} \times \tilde{\mathbf{B}}\|_{\mathbf{P}}^{2}\right) \leq C_{1}\left(\|\tilde{\mathbf{B}}\|_{\mathbf{P}}^{2}+\left(\frac{\delta_{e}}{L_{0}}\right)^{2} \frac{1}{\rho}\|\mathbf{D} \times \tilde{\mathbf{B}}\|_{\mathbf{P}}^{2}\right) .
$$

Since the divergence is not preserved by the symmetric scheme, we show that the divergence generated by the symmetric scheme is bounded (a similar result for the ideal magnetic induction equations was shown in [13]).

Theorem 3.2 Let $\tilde{\mathbf{u}}_{i, j, k}=\mathbf{u}\left(x_{i}, y_{j}, z_{k}\right)$ be the point evaluation of a function $u \in C^{2}$ and let the solutions of (3.11) go to zero at infinity, then the following estimates hold

$$
\frac{d}{d t}\|\mathbf{D} \cdot \tilde{\mathbf{B}}\|_{\mathbf{P}}^{2} \leq C\left(\|\mathbf{D} \cdot \tilde{\mathbf{B}}\|_{\mathbf{P}}^{2}+\|\tilde{\mathbf{B}}\|^{2}\right)
$$

with $C$ a constant that depend on $\mathbf{u}$ and its derivative and on the regularity of the grid.

Proof We define the discrete divergence $\hat{\omega}=\mathbf{D} \cdot \tilde{\mathbf{B}}$ and using the numerical scheme (3.11) with Corollary 3.3, we obtain an equation for its evolution

$$
\frac{d}{d t} \hat{\omega}=\mathbf{D} \cdot(\mathscr{A} \mathscr{V}(\tilde{\mathbf{B}}, \tilde{\mathbf{u}})-(\tilde{\mathbf{u}} \circ \mathbf{D}) \tilde{\mathbf{B}})
$$

Now expanding each component of $\mathscr{A} \mathscr{V}$ and using Lemma 3.1, we obtain, for example for the first component:

$$
\begin{aligned}
(\mathscr{A} \mathscr{V}(\tilde{\mathbf{B}}, \tilde{\mathbf{u}}))^{1}= & \overline{\mathscr{A}}_{y}\left(\tilde{\mathbf{B}}^{2}\right) \circ \mathfrak{d}_{y} \tilde{\mathbf{u}}^{1}+\overline{\mathscr{A}}_{z}\left(\tilde{\mathbf{B}}^{3}\right) \circ \mathfrak{d}_{z} \tilde{\mathbf{u}}^{1} \\
& -\overline{\mathscr{A}}_{y}\left(\tilde{\mathbf{B}}^{1}\right) \circ \mathfrak{d}_{y} \tilde{\mathbf{u}}^{2}-\overline{\mathscr{A}}_{z}\left(\tilde{\mathbf{B}}^{1}\right) \circ \mathfrak{d}_{z} \tilde{\mathbf{u}}^{3}
\end{aligned}
$$




$$
\begin{aligned}
= & \mathfrak{d}_{y}\left(\tilde{\mathbf{B}}^{2} \hat{\mathbf{u}}^{1}-\tilde{\mathbf{B}}^{1} \hat{\mathbf{u}}^{2}\right)-\overline{\mathbf{u}}^{1} \circ \omega+(\tilde{\mathbf{u}} \circ \mathbf{D}) \tilde{\mathbf{B}}^{1}+\mathfrak{R}^{\Delta y}\left(\tilde{\mathbf{B}}^{1}, \tilde{\mathbf{B}}^{2}\right) \\
& +\mathfrak{R}^{\Delta z}\left(\tilde{\mathbf{B}}^{1}, \tilde{\mathbf{B}}^{3}\right),
\end{aligned}
$$

where the residual terms are

$$
\begin{aligned}
& \Re^{\Delta y}\left(\tilde{\mathbf{B}}^{1}, \tilde{\mathbf{B}}^{2}\right)_{i, j, k}=\Delta y \sum_{l=-q}^{q}\left(\gamma_{l}^{j}\left(\tilde{\mathbf{u}}^{2}\right) \tilde{\mathbf{B}}_{i, j+l, k}^{1}-\gamma_{l}^{j}\left(\tilde{\mathbf{u}}^{1}\right) \tilde{\mathbf{B}}_{i, j+l, k}^{2}\right), \\
& \mathfrak{R}^{\Delta z}\left(\tilde{\mathbf{B}}^{1}, \tilde{\mathbf{B}}^{3}\right)_{i, j, k}=\Delta z \sum_{l=-q}^{q}\left(\gamma_{l}^{j}\left(\tilde{\mathbf{u}}^{3}\right) \tilde{\mathbf{B}}_{i, j, k+l}^{1}-\gamma_{l}^{j}\left(\tilde{\mathbf{u}}^{1}\right) \tilde{\mathbf{B}}_{i, j, k+l}^{3}\right) .
\end{aligned}
$$

Here the $\gamma_{k}^{i}$ are defined in the proof of Lemma 3.1 and depend on the velocities $u$. Applying the same technique on the two other components we obtain

$$
(\mathscr{A} \mathscr{V}(\tilde{\mathbf{B}}, \tilde{\mathbf{u}})-(\tilde{\mathbf{u}} \circ \mathbf{D}) \tilde{\mathbf{B}})=(\mathbf{D} \times(\tilde{\mathbf{u}} \times \tilde{\mathbf{B}}))-\tilde{\mathbf{u}} \circ \hat{\omega}+\mathfrak{R}
$$

where

$$
\mathfrak{R}=\left(\begin{array}{c}
\Re^{\Delta y}\left(\tilde{\mathbf{B}}^{1}, \tilde{\mathbf{B}}^{2}\right)+\mathfrak{R}^{\Delta z}\left(\tilde{\mathbf{B}}^{1}, \tilde{\mathbf{B}}^{3}\right) \\
\mathfrak{R}^{\Delta x}\left(\tilde{\mathbf{B}}^{2}, \tilde{\mathbf{B}}^{1}\right)+\mathfrak{R}^{\Delta z}\left(\tilde{\mathbf{B}}^{2}, \tilde{\mathbf{B}}^{3}\right) \\
\mathfrak{R}^{\Delta x}\left(\tilde{\mathbf{B}}^{3}, \tilde{\mathbf{B}}^{1}\right)+\mathfrak{R}^{\Delta y}\left(\tilde{\mathbf{B}}^{3}, \tilde{\mathbf{B}}^{2}\right)
\end{array}\right)
$$

Here $\|\Re\|_{\mathbf{P}} \leq C \max (\Delta x, \Delta y, \Delta z)\|\tilde{\mathbf{B}}\|_{\mathbf{P}}$. Setting these results in the discrete evolution equation an using Corollary 3.3 we obtain

$$
\frac{d}{d t} \hat{\omega}=-\mathbf{D} \cdot(\hat{\omega} \circ \tilde{\mathbf{u}})+\mathbf{D} \Re .
$$

The time evolution of the $\mathbf{P}$ norm of the divergence is

$$
\frac{d}{d t}\|\bar{\omega}\|_{\mathbf{P}}^{2}=2\left(\bar{\omega}, \frac{d}{d t} \bar{\omega}\right)_{\mathbf{P}}=-2(\bar{\omega}, \mathbf{D} \cdot(\bar{\omega} \circ \tilde{\mathbf{u}}))_{\mathbf{P}}+(\bar{\omega}, \mathbf{D} \Re)_{\mathbf{P}}
$$

with summation by parts and Lemma 3.4 we obtain

$$
\begin{aligned}
\frac{d}{d t}\|\bar{\omega}\|_{\mathbf{P}}^{2} & =2(\mathbf{D} \bar{\omega}, \bar{\omega} \circ \tilde{\mathbf{u}})_{\mathbf{P}}+(\bar{\omega}, \mathbf{D} \Re)_{\mathbf{P}}=2(\tilde{\mathbf{u}} \mathbf{D} \circ \bar{\omega}, \bar{\omega})_{\mathbf{P}}+(\bar{\omega}, \mathbf{D} \Re)_{\mathbf{P}} \\
& =(\tilde{\mathbf{u}} \mathbf{D} \circ \bar{\omega}, \bar{\omega})_{\mathbf{P}}-(\mathbf{D} \cdot(\tilde{\mathbf{u}} \circ \bar{\omega}), \bar{\omega})_{\mathbf{P}}+(\bar{\omega}, \mathbf{D} \Re)_{\mathbf{P}} \leq C\left\|_{\bar{\omega}}\right\|_{\mathbf{P}}^{2}+\|\bar{\omega}\|_{\mathbf{P}} \|_{\mathbf{D} \Re \|_{\mathbf{P}}} \\
& \leq C\|\bar{\omega}\|_{\mathbf{P}}^{2}+\frac{C}{\min (\Delta x, \Delta y, \Delta z)}\|\bar{\omega}\|_{\mathbf{P}}\|\Re\|_{\mathbf{P}} \\
& \leq C_{1}\|\bar{\omega}\|_{\mathbf{P}}^{2}+C_{2} \frac{\max (\Delta x, \Delta y, \Delta z)}{\min (\Delta x, \Delta y, \Delta z)}\|\bar{\omega}\|_{\mathbf{P}}\|\tilde{\mathbf{B}}\|_{\mathbf{P}},
\end{aligned}
$$

where the $C$ 's depend on $u$ and its derivative. To obtain this result the boundary terms are neglected since $\tilde{\mathbf{B}}$ decays to 0 at infinity. We have also used that

$$
(\hat{x}, \tilde{\mathbf{v}} \circ \hat{y})_{\mathbf{P}}=(\tilde{\mathbf{v}} \circ \hat{x}, \hat{y})_{\mathbf{P}}
$$


where $\hat{x}$ and $\hat{y}$ are scalar grid functions and $\tilde{\mathbf{v}}$ a vector grid function. This is the case since $P$ is a diagonal matrix. We conclude the proof using Cauchy's inequality.

Although the symmetric scheme (3.11) is stable in $H^{1}$, it might generate bounded divergence errors. Next, we design a scheme that preserves a discrete version of the divergence operator.

\subsection{Divergence preserving schemes}

The symmetric scheme does not preserve a discrete version of the divergence constraint as it discretizes the symmetric version (2.2) of the Magnetic induction equations with Hall effect. We have to discretize the non-symmetric standard version (1.12) of the Magnetic induction equations with Hall effect in order to design a divergence preserving scheme. Such a scheme for is

$$
\begin{aligned}
\frac{d}{d t}\left(\tilde{\mathbf{B}}_{i, j, k}+\left(\frac{\delta_{e}}{L_{0}}\right)^{2} \frac{1}{\rho}(\mathbf{D} \times \mathbf{D} \times \tilde{\mathbf{B}})_{i, j, k}\right) \\
=\mathbf{D} \times\left(\tilde{\mathbf{u}} \times \tilde{\mathbf{B}}_{i, j, k}\right)-\eta\left(\mathbf{D} \times\left(\mathbf{D} \times \tilde{\mathbf{B}}_{i, j, k}\right)\right) \\
-\left(\frac{\delta_{e}}{L_{0}}\right)^{2} \frac{1}{\delta} \mathbf{D} \times\left(\left(\tilde{\mathbf{u}}_{i, j, k} \cdot \mathbf{D}\right)\left(\mathbf{D} \times \tilde{\mathbf{B}}_{i, j, k}\right)\right) \\
-\frac{\delta_{i}}{L_{0}} \frac{1}{\delta} \mathbf{D} \times\left(\left(\mathbf{D} \times \tilde{\mathbf{B}}_{i, j, k}\right) \times \tilde{\mathbf{B}}_{i, j, k}\right) .
\end{aligned}
$$

The application of Corollary 3.3 shows that the above scheme clearly preserve the divergence:

$$
\frac{d}{d t} \mathbf{D} \cdot \tilde{\mathbf{B}}=0
$$

That means that if we have a initial data with zero discrete divergence the divergence constraint will be satisfied.

The proof for the energy stability of this scheme is more complex since the equation is not symmetric. We introduce the following one-sided operators:

$$
D_{x}^{s} w_{i}=\frac{w_{i+s}-w_{i}}{s \Delta x} .
$$

The operators $D_{y}^{s}$ and $D_{z}^{s}$ are defined analogously.

Lemma 3.6 For two grid functions $u$ and $w$, the following identity holds,

$$
D_{x}(u \circ w)=u \circ D_{x} w+\hat{A}_{x}\left(\left(D_{x}^{s} u\right), w\right),
$$

with $\hat{A}_{x}\left(\left(D_{x}^{s} u\right), w\right)_{i}=\sum_{k=-q}^{q} k \beta_{k}\left(D_{x}^{k} u_{i}\right) w_{i+k}$ is an average over discrete one sided derivative of $u$ multiplied with $w$. 
Proof We compute the difference

$$
\begin{aligned}
D_{x}(u \circ w)_{i}-u \circ D_{x} w & =\frac{1}{\Delta x} \sum_{k=-q}^{q} \beta_{k} u_{i+k} w_{i+k}-u_{i} \frac{1}{\Delta x} \sum_{k=-q}^{q} \beta_{k} w_{i+k} \\
& =\frac{1}{\Delta x} \sum_{k=-q}^{q} \beta_{k} k \frac{u_{i+k}-u_{i}}{k \Delta x} w_{i+k},
\end{aligned}
$$

noting that it takes the form of the desired average.

The energy bound for the divergence preserving scheme is given below:

Theorem 3.3 Let $\tilde{\mathbf{u}}_{i, j, k}=\mathbf{u}\left(x_{i}, y_{j}, z_{k}\right)$ be the point evaluation of a function $u \in C^{2}$ and let the solutions of (3.19) with an initial data with $\mathbf{D} \cdot \tilde{\mathbf{B}}_{0}=0$. If the approximate solution decays to zero at infinity, then the following estimates hold

$$
\frac{d}{d t}\left(\|\tilde{\mathbf{B}}\|_{\mathbf{P}}^{2}+\left(\frac{\delta_{e}}{L_{0}}\right)^{2} \frac{1}{\rho}\|\mathbf{D} \times \tilde{\mathbf{B}}\|_{\mathbf{P}}^{2}\right) \leq C\left(\|\tilde{\mathbf{B}}\|_{\mathbf{P}}^{2}+\left(\frac{\delta_{e}}{L_{0}}\right)^{2} \frac{1}{\rho}\|\mathbf{D} \times \tilde{\mathbf{B}}\|_{\mathbf{P}}^{2}\right)
$$

with $C$ a constant that depends on $\mathbf{u}$ and its derivative only.

Proof To prove the energy estimate, we have to symmetrize the advection terms in the scheme. This is possible since the method preserve divergence; in this case we can subtract form (3.19) $\tilde{\mathbf{v}}(\mathbf{D} \cdot \tilde{\mathbf{B}})=0$.

Using Lemma 3.6, we cam reformulate the discrete advection part

$$
\mathbf{D} \times(\tilde{\mathbf{u}} \times \tilde{\mathbf{B}})-\tilde{\mathbf{v}}(\mathbf{D} \cdot \tilde{\mathbf{B}})=-(\tilde{\mathbf{u}} \cdot \mathbf{D}) \tilde{\mathbf{B}}+\mathscr{R}(\tilde{\mathbf{B}}, \tilde{\mathbf{u}}),
$$

with

$$
\begin{aligned}
\mathscr{R}(\tilde{\mathbf{B}}, \tilde{\mathbf{u}})_{i, j, k} & \\
= & \left(\begin{array}{l}
\mathscr{A}_{y}\left[\left(\mathfrak{d}_{y}^{s}\left(\hat{u}_{1}\right) \hat{B}_{2}\right)_{i, j, k}-\left(\mathfrak{d}_{y}^{s}\left(\hat{u}_{2}\right) \hat{B}_{1}\right)_{i, j, k}\right]+\mathscr{A}_{z}\left[\left(\mathfrak{d}_{z}^{s}\left(\hat{u}_{1}\right) \hat{B}_{3}\right)_{i, j, k}-\left(\mathfrak{d}_{z}^{s}\left(\hat{u}_{3}\right) \hat{B}_{1}\right)_{i, j, k}\right] \\
\mathscr{A}_{x}\left[\left(\mathfrak{d}_{y}^{s}\left(\hat{u}_{2}\right) \hat{B}_{1}\right)_{i, j, k}-\left(\mathfrak{d}_{x}^{s}\left(\hat{u}_{1}\right) \hat{B}_{2}\right)_{i, j, k}\right]+\mathscr{A}_{z}\left[\left(\mathfrak{d}_{z}^{s}\left(\hat{u}_{2}\right) \hat{B}_{3}\right)_{i, j, k}-\left(\mathfrak{d}_{z}^{s}\left(\hat{u}_{3}\right) \hat{B}_{2}\right)_{i, j, k}\right] \\
\mathscr{A}_{x}\left[\left(\mathfrak{d}_{x}^{s}\left(\hat{u}_{3}\right) \hat{B}_{1}\right)_{i, j, k}-\left(\mathfrak{d}_{x}^{s}\left(\hat{u}_{1}\right) \hat{B}_{3}\right)_{i, j, k}\right]+\mathscr{A}_{y}\left[\left(\mathfrak{d}_{y}^{s}\left(\hat{u}_{3}\right) \hat{B}_{2}\right)_{i, j, k}-\left(\mathfrak{d}_{y}^{s}\left(\hat{u}_{2}\right) \hat{B}_{3}\right)_{i, j, k}\right]
\end{array}\right) .
\end{aligned}
$$

The resulting scheme is

$$
\begin{aligned}
\frac{d}{d t}\left[\tilde{\mathbf{B}}_{i, j, k}+\left(\frac{\delta_{e}}{L_{0}}\right)^{2} \frac{1}{\rho}(\mathbf{D} \times \mathbf{D} \times \tilde{\mathbf{B}})_{i, j, k}\right] \\
=-(\tilde{\mathbf{u}} \cdot D) \tilde{\mathbf{B}}-\eta\left(\mathbf{D} \times\left(\mathbf{D} \times \tilde{\mathbf{B}}_{i, j, k}\right)\right) \\
\quad-\left(\frac{\delta_{e}}{L_{0}}\right)^{2} \frac{1}{\delta} \mathbf{D} \times\left(\left(\tilde{\mathbf{u}}_{i, j, k} \cdot \mathbf{D}\right)\left(\mathbf{D} \times \tilde{\mathbf{B}}_{i, j, k}\right)\right) \\
\quad-\frac{\delta_{i}}{L_{0}} \frac{1}{\delta} \mathbf{D} \times\left(\left(\mathbf{D} \times \tilde{\mathbf{B}}_{i, j, k}\right) \times \tilde{\mathbf{B}}_{i, j, k}\right)+\mathscr{R}(\tilde{\mathbf{u}}, \tilde{\mathbf{B}}) .
\end{aligned}
$$


We see that this is very similar to result obtained for the symmetric case. The only difference is that, instead the average term $(\tilde{\mathbf{B}}, \mathscr{A} \mathscr{V}(\tilde{\mathbf{B}}, \mathbf{u}))$ p we have a residual term $(\tilde{\mathbf{B}}, \mathscr{R}(\tilde{\mathbf{B}}, \mathbf{u}))_{\mathbf{P}}$. Then showing that the residual term is bounded

$$
(\tilde{\mathbf{B}}, \mathscr{R}(\tilde{\mathbf{B}}, \mathbf{u}))_{\mathbf{P}} \leq C\|\tilde{\mathbf{B}}\|_{\mathbf{P}}^{2}
$$

will conclude the proof. We can follow the same procedure we used in the proof of Lemma 3.5 to bound the advection term.

The proof follows the theory presented in [14] where a generalized finite volume scheme that preserve discrete divergence is presented. The method presented there is more general in its formulation, and includes a large class of already known divergence preserving methods.

\subsection{Time stepping}

Both the semi-discrete symmetric scheme and divergence preserving schemes can be written as

$$
\frac{d}{d t}(\hat{\mathbf{B}}+\alpha \mathbf{D} \times \mathbf{D} \times \hat{\mathbf{B}})=\mathscr{G}(\hat{\mathbf{B}}, \hat{\mathbf{u}}),
$$

with $\alpha=\left(\frac{\delta_{e}}{L_{0}}\right)^{2} \frac{1}{\rho}$ and the function $\mathscr{G}$ will depend on the scheme.

We use a standard Runge Kutta method to update the solution in time. Even though we use explicit RK methods, we have to solve linear equations (corresponding to the lhs of the above scheme) at each time step. As an example, we consider a second order SSP method ([5]):

$$
\begin{aligned}
\hat{\mathbf{B}}^{\star} & =\hat{\mathbf{B}}^{n}+\Delta t A^{-1} \mathscr{G}\left(\hat{\mathbf{B}}^{n}, \hat{\mathbf{u}}\right), \\
\hat{\mathbf{B}}^{\star \star} & =\hat{\mathbf{B}}^{\star}+\Delta t A^{-1} \mathscr{G}\left(\hat{\mathbf{B}}^{\star}, \hat{\mathbf{u}},\right) \\
\hat{\mathbf{B}}^{n+1} & =\frac{\hat{\mathbf{B}}^{n}+\hat{\mathbf{B}}^{\star \star}}{2} .
\end{aligned}
$$

Here, $A=I+\alpha F, F$ is the matrix representation of $\mathbf{D} \times \mathbf{D}$ and $I$ is the identity matrix. In this paper, we are using a direct solver in each time sub-step.

Remark 3.1 The main aim of this paper is to present a robust spatial discretization. The time stepping that we use is fairly standard. Moreover, any time-stepping procedure for equations involving mixed spatio-temporal derivatives requires a robust implicit solver. In our case, the matrices $F$ and $A$ are of size $3 \times N_{x} \times N_{y} \times N_{z}$, and are ill-conditioned. The key issue in designing efficient time stepping procedures for the magnetic induction equations is to design a suitable preconditioner. This is complicated due to the saddle point type structure of the Maxwell's equations. Recently, robust preconditioners have been developed for Maxwell type problems that use finite element type discretizations [7] and references therein. Our discretization, being a finite difference discretization, does not satisfy the requirements of this theory. The design of an efficient preconditioner for the magnetic induction equations with Hall effect goes beyond the scope of this paper and is the subject of ongoing research. 


\section{Numerical experiments}

For simplicity, we consider the Magnetic induction equations with Hall effect in two space dimensions and present numerical experiments comparing different schemes proposed above. In two space dimensions, the symmetric version (2.2) reads as

$$
\begin{aligned}
\frac{\partial}{\partial t}[ & \left.\hat{\mathbf{B}}+\left(\frac{\delta_{e}}{L_{0}}\right)^{2} \frac{1}{\rho} \widehat{\nabla} \times \widehat{\nabla} \times \hat{\mathbf{B}}\right] \\
= & \hat{C_{1}} \hat{\mathbf{B}}-(\hat{\mathbf{u}} \cdot \nabla) \hat{\mathbf{B}}-\eta \widehat{\nabla} \times \widehat{\nabla} \times \hat{\mathbf{B}} \\
& \quad-\left(\frac{\delta_{e}}{L_{0}}\right)^{2} \frac{1}{\rho} \widehat{\nabla} \times((\hat{\mathbf{u}} \cdot \nabla)(\widehat{\nabla} \times \hat{\mathbf{B}}))-\frac{\delta_{i}}{L_{0}} \frac{1}{\rho} \widehat{\nabla} \times\left(\hat{\mathbf{B}} \cdot \nabla B_{3}\right), \\
\frac{\partial}{\partial t}[ & \left.B_{3}-\left(\frac{\delta_{e}}{L_{0}}\right)^{2} \frac{1}{\rho} \Delta B_{3}\right] \\
= & \mathbf{C}_{2} \mathbf{B}-\hat{\mathbf{u}} \nabla B_{3}+\eta \Delta B_{3}+\left(\frac{\delta_{e}}{L_{0}}\right)^{2} \frac{1}{\rho} \nabla \cdot\left((\hat{\mathbf{u}} \cdot \nabla) \nabla B_{3}\right) \\
& -\frac{\delta_{i}}{L_{0}} \frac{1}{\rho} \nabla \cdot(\hat{\mathbf{B}} \cdot(\widehat{\nabla} \times \hat{\mathbf{B}})) .
\end{aligned}
$$

Here, $\hat{\mathbf{B}}=\left(B_{1}, B_{2}\right)^{\top}$ and $\hat{\mathbf{u}}=\left(u_{1}, u_{2}\right)^{\top}$. We have also introduced a compact "curl" operator $\widehat{\nabla} \times$ in two dimensions:

$$
\begin{aligned}
\widehat{\nabla} \times\left(\begin{array}{c}
v_{1} \\
v_{2}
\end{array}\right):=\frac{\partial v_{2}}{\partial x}-\frac{\partial v_{1}}{\partial y}, \\
\widehat{\nabla} \times \psi:=\left(\begin{array}{c}
\frac{\partial \psi}{\partial y} \\
-\frac{\partial \psi}{\partial x}
\end{array}\right),
\end{aligned}
$$

where $\hat{\mathbf{v}}: \mathbb{R}^{3} \rightarrow \mathbb{R}^{2}$ and $\psi: \mathbb{R}^{3} \rightarrow \mathbb{R}$.

\subsection{Smooth problems}

\subsubsection{Pure magnetic advection}

First, we test the proposed numerical schemes for the magnetic induction equations without Hall, electron inertia and resistivity terms. We take the velocity field

$$
\mathbf{u}=(-y, x)^{\top}
$$

in the following. Then, (4.1a), (4.1b) with $\eta=\delta_{i}=\delta_{e}=0$ has an exact solution (see [3]) given by

$$
\hat{\mathbf{B}}(x, y, y)=R(t) \hat{\mathbf{B}}_{0}(R(-t)(x, y)),
$$

with $R(t)$ a rotation matrix with angle $t$. 


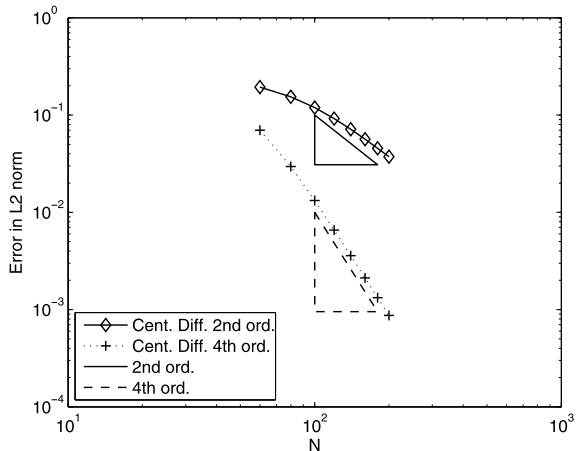

(a) Symmetric schemes

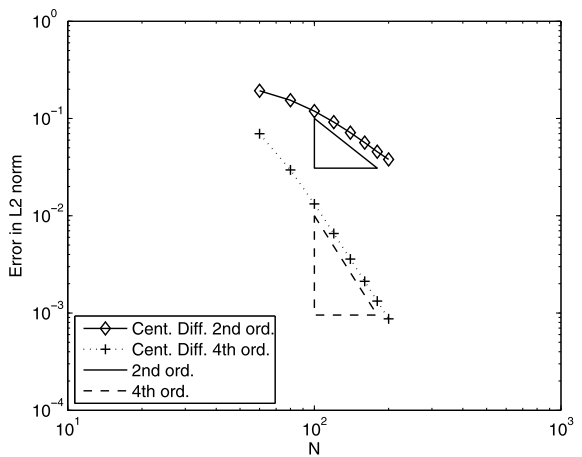

(b) Divergence preserving schemes

Fig. 1 Convergence plots for the advection problem for the different schemes analyzed

Table 1 Convergence rates for the magnetic advection problem for different schemes

\begin{tabular}{lll}
\hline & 2nd ord & 4 ord \\
\hline Preserving & 1.89 & 3.98 \\
Symmetric & 1.89 & 3.98 \\
\hline
\end{tabular}

The initial data is

$$
\hat{\mathbf{B}}_{0}(x, y)=4\left(\begin{array}{c}
-y \\
x-\frac{1}{2}
\end{array}\right) e^{-20\left((x-1 / 2)^{2}+y^{2}\right)}
$$

in the computational domain $\Omega=[-2.5,2.5] \times[-2.5,2.5]$. We consider Neumann type non-reflecting boundary conditions. The exact solution represents the rotation of the initial hump around the domain with the hump completing one rotation in the period $T=2 \pi$.

We will test the following four schemes: the second- and fourth-order versions of the symmetric scheme (3.11) with difference operators given in appendix $A$, secondand fourth-order version of the divergence preserving scheme (3.19). The convergence plots in $L^{2}$ are shown in Fig. 1 .

For time integration we have used a second order SSP and a standard fourth order Runge-Kutta method. The results are obtained using different mesh sizes, form 60 to 200 points. The experimental convergence orders are shown in Table 1 and demonstrate that the expected orders of accuracy are obtained in practice.

\subsubsection{Forced solutions}

In order to test the convergence rates for various schemes for the full Magnetic induction equations with Hall effect, we add a forcing term such that the rotating hump (4.3) remains a solution of the forced equations. The Hall induction equations with the forcing term are

$$
\frac{\partial}{\partial t}\left[\hat{\mathbf{B}}+\left(\frac{\delta_{e}}{L_{0}}\right)^{2} \frac{1}{\rho} \widehat{\nabla} \times \widehat{\nabla} \times \hat{\mathbf{B}}\right]
$$




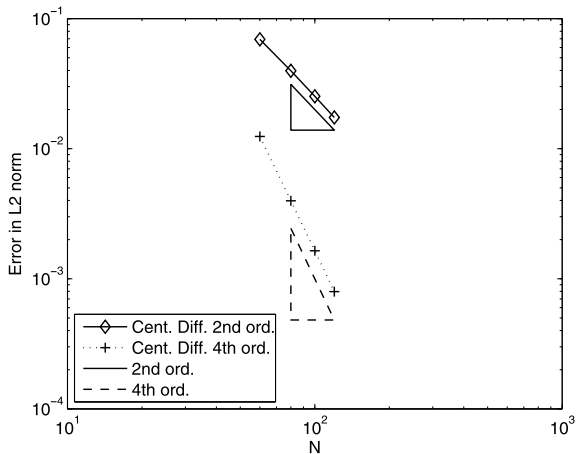

(a) Symmetric schemes

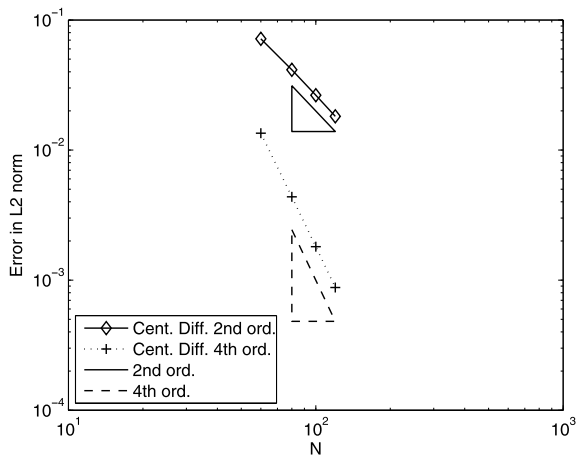

(b) Divergence preserving schemes

Fig. 2 Convergence plots for the Forced Problem with $\eta=0.01, \delta_{i}=0.01$ and $\delta_{e}=4.5 \times 10^{-2}$

Table 2 Convergence rates for the Forced Problem with $\eta=0.01, \delta_{i}=0.01$ and $\delta_{e}=4.5 \times 10^{-2}$

\begin{tabular}{lll}
\hline & 2nd ord & 4 ord \\
\hline Preserving & 2.05 & 3.97 \\
Symmetric & 2.05 & 3.98 \\
\hline
\end{tabular}

$$
\begin{aligned}
= & \hat{C}_{1} \hat{\mathbf{B}}-(\hat{\mathbf{u}} \cdot \nabla) \hat{\mathbf{B}}-\eta \widehat{\nabla} \times \widehat{\nabla} \times \hat{\mathbf{B}} \\
& -\left(\frac{\delta_{e}}{L_{0}}\right)^{2} \frac{1}{\rho} \widehat{\nabla} \times((\hat{\mathbf{u}} \cdot \nabla)(\widehat{\nabla} \times \hat{\mathbf{B}})) \\
& -\frac{\delta_{i}}{L_{0}} \frac{1}{\rho} \widehat{\nabla} \times\left(\hat{\mathbf{B}} \cdot \nabla B_{3}\right)+\hat{\mathbf{S}}(x, y, t) \\
\frac{\partial}{\partial t}[ & \left.B_{3}-\left(\frac{\delta_{e}}{L_{0}}\right)^{2} \frac{1}{\rho} \Delta B_{3}\right]=\mathbf{C}_{2} \mathbf{B}-\hat{\mathbf{u}} \nabla B_{3}+\eta \Delta B_{3} \\
& +\left(\frac{\delta_{e}}{L_{0}}\right)^{2} \frac{1}{\rho} \nabla \cdot\left((\hat{\mathbf{u}} \cdot \nabla) \nabla B_{3}\right)-\frac{\delta_{i}}{L_{0}} \frac{1}{\rho} \nabla \cdot(\hat{\mathbf{B}} \cdot(\widehat{\nabla} \times \hat{\mathbf{B}}))+S^{3}(x, y, t)
\end{aligned}
$$

The forcing term $\hat{\mathbf{S}}$ is

$$
\begin{aligned}
\hat{\mathbf{S}}(x, y, t)= & 160 P(x, y, t) \eta e^{-20\left((x \cos (t)+y \sin (t)-1 / 2)^{2}+(y \cos (t)-x \sin (t))^{2}\right.} \\
& \times\left(\begin{array}{c}
\sin (t)-2 y \\
2 x-\cos (t)
\end{array}\right) \\
S^{3}(x, y, t)= & 0 .
\end{aligned}
$$

Here $P(x, y, t)=20 x \cos (t)+20 y \sin (t)-20 x^{2}-20 y^{2}-3$.

The convergence results for four different schemes are presented in Fig. 2. The obtained orders of convergence are shown in Table 2. Again, the expected orders of convergence are obtained. 
Table 3 Discrete divergence for the Hall problem

\begin{tabular}{lll}
\hline & Symmetric & Preserving \\
\hline 25 & $4.6 e-2$ & $6.4 e-17$ \\
50 & $2.4 e-5$ & $8.6 e-17$ \\
75 & $6.2 e-7$ & $9.1 e-17$ \\
100 & $2.5 e-7$ & $1.1 e-16$ \\
125 & $1.4 e-7$ & $1.2 e-16$ \\
\hline
\end{tabular}

\subsection{Hall effect solutions}

Next, we test the full Magnetic induction equations with Hall effect(without any forcing) for the rotating hump problem. We set $\eta=0.01, \delta_{i}=0.1$ and $\delta_{e}=4.5 \times 10^{-2}$ and compute the solutions on a mesh $160 \times 160$ points.

We compare the results with those obtained for the pure advection of the hump.

The results are shown in Fig. 3 and demonstrate the robustness of the secondorder symmetric scheme. Similar results were obtained with the divergence preserving scheme. The results show that the addition of resistivity, electron inertia and Hall effect leads to diffusion of the original hump and the creation of a non-zero $B_{3}$ component even if the initial $B_{3}$ is set to zero.

We conclude by tabulating the discrete divergence generated by the schemes for this problem in Table 3. As expected, the divergence preserving scheme preserves divergence to machine precision. On the other hand, the symmetric scheme does generate some spurious divergence.

The divergence errors converge quite rapidly to zero as the mesh is refined. Furthermore, there was no noticeable difference in the quality of the results for the primary solution variables between the symmetric and divergence preserving schemes.

Remark 4.1 An interesting issue that appears in the context of numerical approximation of evolution equations like the magnetic induction equations with Hall effect is the issue of long time behavior of the numerical scheme. In order to test this behavior for our proposed schemes, we simulate three full rotations $(T=6 \pi)$ with the above set up (no forcing) and present the results in Fig. 4. As shown in the figure, the initial hump is dissipated to some extent, by the time it completes three full rotations. Most of this dissipation is physical and is caused by the magnetic resistivity term in the magnetic induction equations with Hall effect. However, some of the dissipation will be on account of the implicit numerical dissipation introduced by the scheme. The loss of magnetic energy (on account of magnetic resistivity as well as some numerical diffusion) is best shown in the energy plot of Fig. 4 (Bottom Left) and documents that a considerable amount of energy is lost by the time the initial hump completes about five full rotations. Hence, the long time behavior of the solution (as well as the scheme) may not be of much interest for these equations as the magnetic resistivity will lead to considerable energy loss and decay of the solutions. In any case, using high-order schemes, high-order time integration routines and suitable implementation of boundary conditions will minimize the numerical dissipation and improve the long time asymptotic properties of the proposed numerical schemes. 


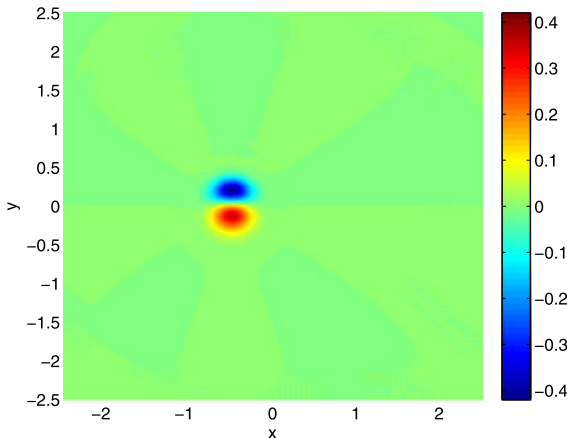

(a) $B_{1}$ component for the magnetic advection problem.

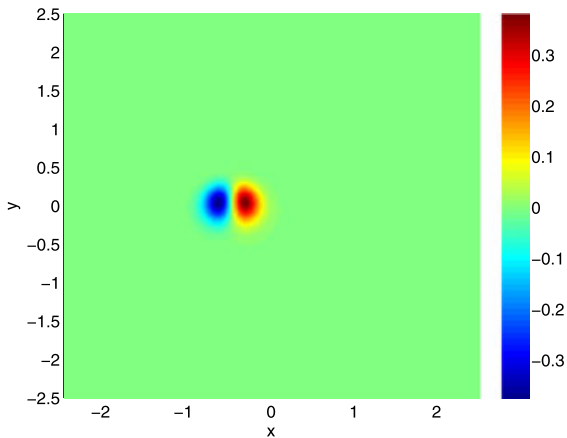

(c) $B_{2}$ component for the magnetic advection problem.

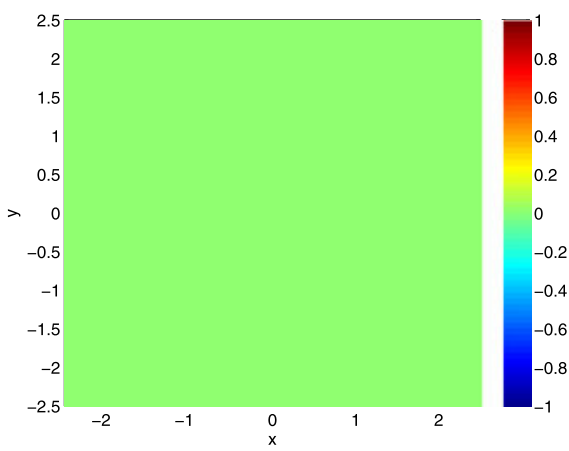

(e) $B_{3}$ component for the magnetic advection problem.

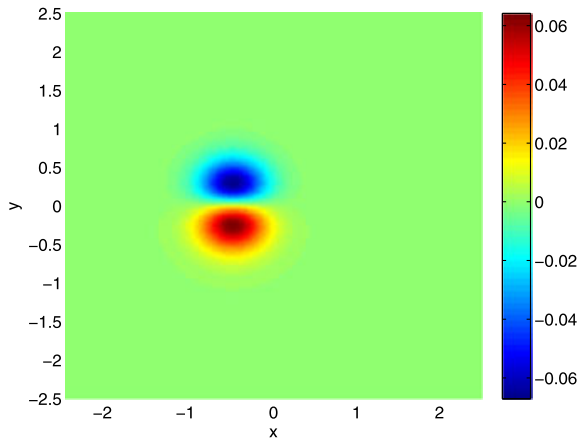

(b) $B_{1}$ component for the Hall problem.

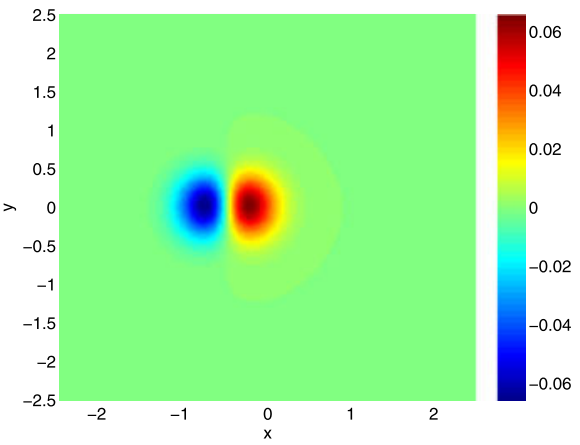

(d) $B_{2}$ component for the Hall problem.

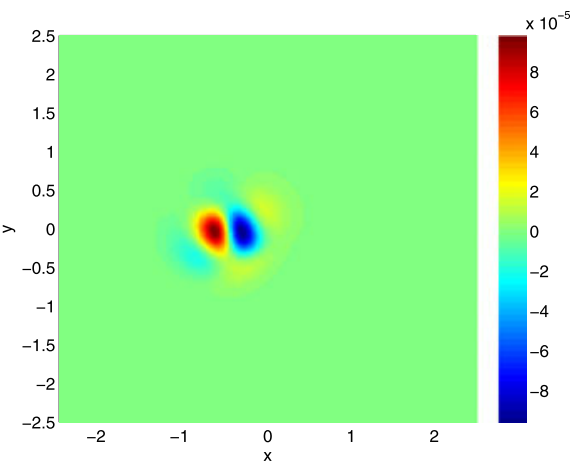

(f) $B_{3}$ component for the Hall problem.

Fig. 3 On the right plots for solution with $\eta=0.01, \delta_{i}=0.1$ and $\delta_{e}=4.5 \times 10^{-2}$ after $T=\pi$ and on the left the advected solution after the same time 


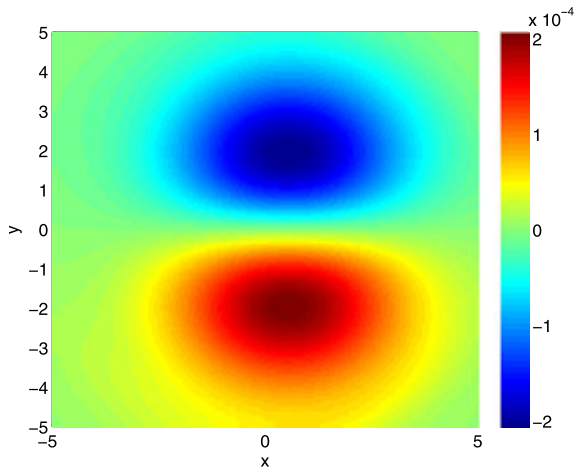

(a) $B_{1}$ after 3 rotations.

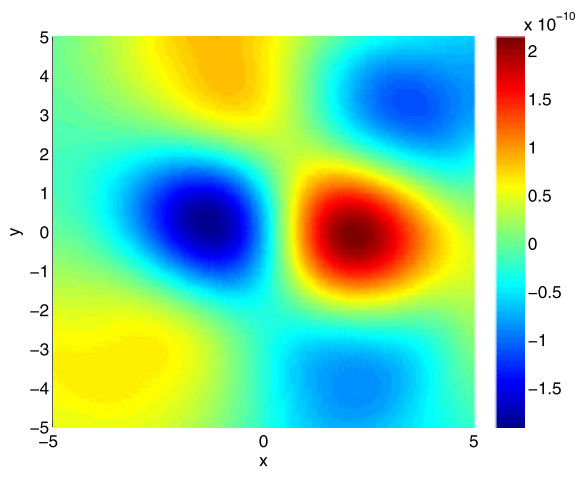

(c) $B_{3}$ after 3 rotations.

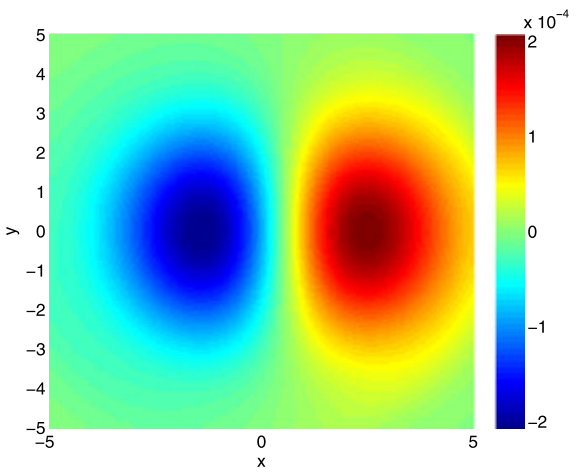

(b) $B_{2}$ after 3 rotations.

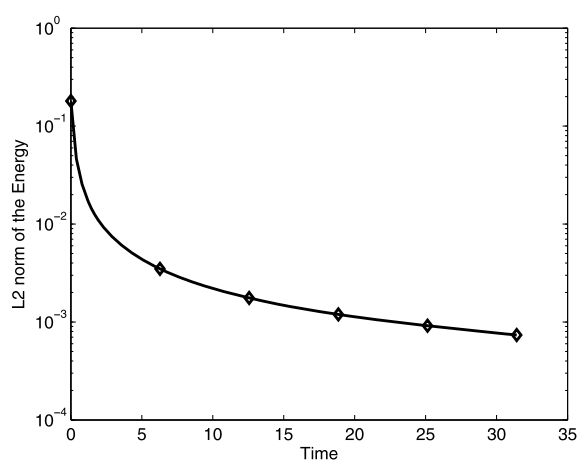

(d) Energy dissipation in time, the marks represents one full rotation.

Fig. 4 Solution on the domain $\Omega=[-5,5]^{2}$ with $\eta=0.01, \delta_{i}=0.01$ and $\delta_{e}=4.5 \times 10^{-2}$ after $T=6 \pi$. On the bottom right corner we have the evolution of discrete energy $\|\tilde{\mathbf{B}}\|_{\mathbf{P}}+\left(\delta_{e} / L_{0}\right)^{2} / \rho\|\mathbf{D} \times \tilde{\mathbf{B}}\|_{\mathbf{P}}$ over five rotations

\subsection{Discontinous problems}

The above results showed that both the symmetric as well as divergence preserving schemes worked very well for smooth problems. There was little to distinguish them. As commented before, the full MHD equations with Hall effect will contain discontinuities in the form of shock waves. We mimic this effect at the level of the magnetic induction equations with Hall effect by considering a discontinuous velocity field (note that the plasma velocities in MHD can be discontinuous):

$$
\hat{\mathbf{u}}(x, y)= \begin{cases}\left(\begin{array}{l}
1 \\
1
\end{array}\right) & \text { for }-\frac{6}{5} \leq x \leq \frac{6}{5} \\
\left(\begin{array}{c}
1 \\
1 / 2
\end{array}\right) & \text { elsewhere }\end{cases}
$$


We take an smooth initial magnetic fields with compact support

$$
\begin{aligned}
& \hat{\mathbf{B}}(x, y)=100\left(\begin{array}{c}
y \\
-x
\end{array}\right)\left\{\begin{array}{lc}
e^{-\frac{2}{1-x^{2}-y^{2}}} & \text { for } x^{2}+y^{2} \leq 0, \\
0 & \text { elsewhere }
\end{array}\right. \\
& B_{3}(x, y)=0.0
\end{aligned}
$$

over a domain $\Omega=[-2.5,2.5] \times[-2.5,2.5]$ with double periodic boundary condition. We choose $\eta=0.5, \delta_{i}=0.2$ and $\delta_{e}=0.3162$ for a mesh of $200 \times 200$ cells and run a simulations until $T=0.4$. We will use both schemes, the divergence preserving and the symmetric one. In the absence of analytical solutions, we will compare the two schemes on a fixed mesh.

Comparing the two schemes in Fig. 5, we note that the symmetric scheme is more oscillatory than the divergence preserving scheme. These oscillations are small and can be seen only in the third component of the solution.

It is essential to point out that our theoretical stability results required that the underlying velocity field be smooth, at least $C^{2}$. Although the velocity field in this experiment is discontinuous, the divergence preserving scheme resolves the solution quite well, indicating its robustness on a challenging test problem. The symmetric scheme is more oscillatory on this test case, indicating that the divergence preserving scheme adds a greater amount of diffusion than the symmetric scheme.

\section{Conclusion}

The MHD equations with Hall effect have been considered as a suitable model for many interesting phenomena in plasma physics, particularly fast magnetic reconnection. These equations are very complicated to approximate numerically as they are multi-dimensional, highly nonlinear, contain possibly discontinuous solutions and involve third order spatial and mixed spatio-temporal derivatives.

Our strategy to numerically approximate these equations involves two steps: in the first step (considered in this paper), we study a relevant sub-model: the magnetic induction equations with Hall effect. These equations contain all the relevant physics in terms of electromagnetics, namely magnetic induction, the presence of resistivity and the inclusion of the nonlinear hall term and high order electron inertia term. In particular, this sub-model serves as a test bed to design efficient discretizations of the magnetic resistivity and of the Hall and electron inertia terms. Here, we have proposed two efficient numerical schemes to approximate the magnetic induction equations with Hall effect. One of the schemes is based on the symmetric formulation of the problem and shown to be energy stable. However, it may not respect a discrete version of the divergence constraint. The second scheme that we propose is designed to preserve a discrete version of the divergence constraint. We were also able to show that this scheme satisfies the relevant energy estimates. Both schemes were tested on series of numerical experiments are shown to perform satisfactorily. For problems with discontinuous velocity fields, the divergence preserving was shown to slightly superior. 


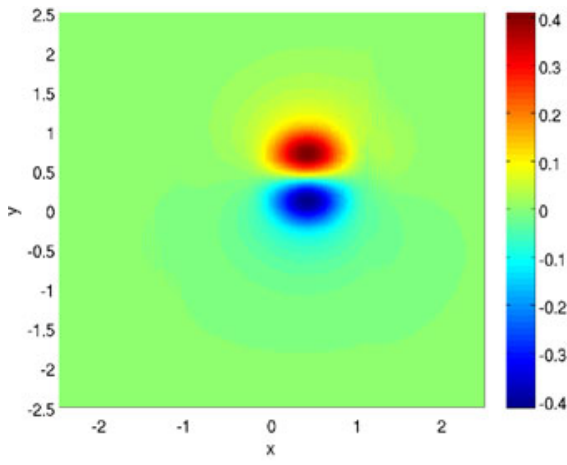

(a) $B_{1}$ component for the symmertic scheme.

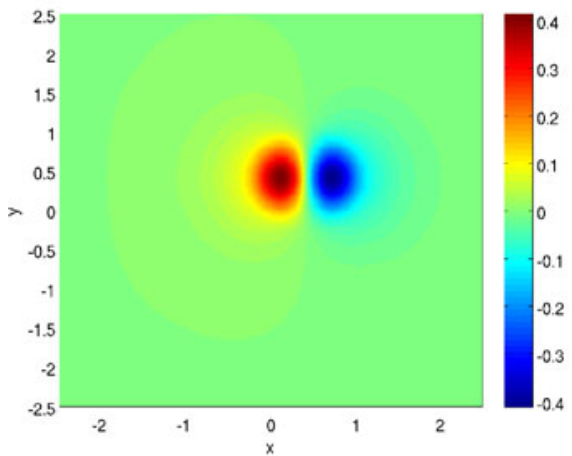

(c) $B_{2}$ component for the symmertic scheme.

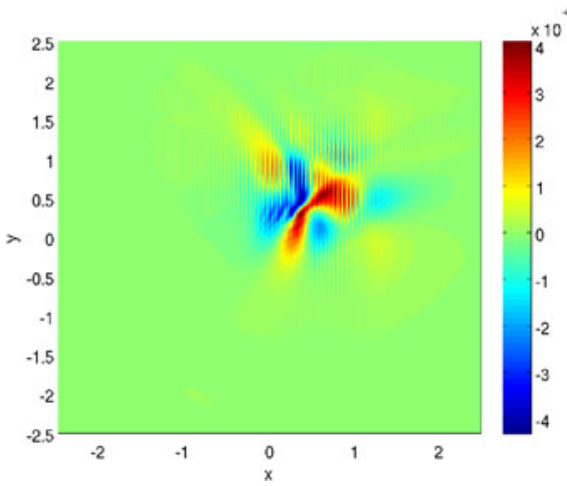

(e) $B_{3}$ component the symmertic scheme.

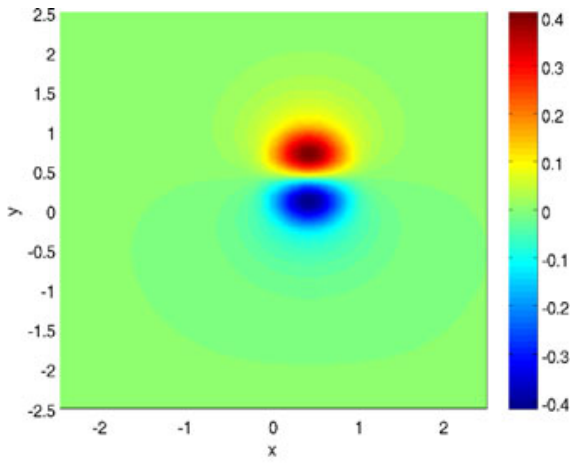

(b) $B_{1}$ component for the divergence preserving scheme.

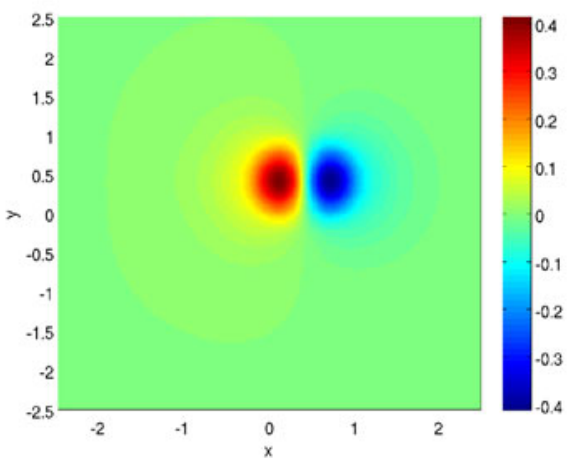

(d) $B_{2}$ component for the divergence preserving scheme.

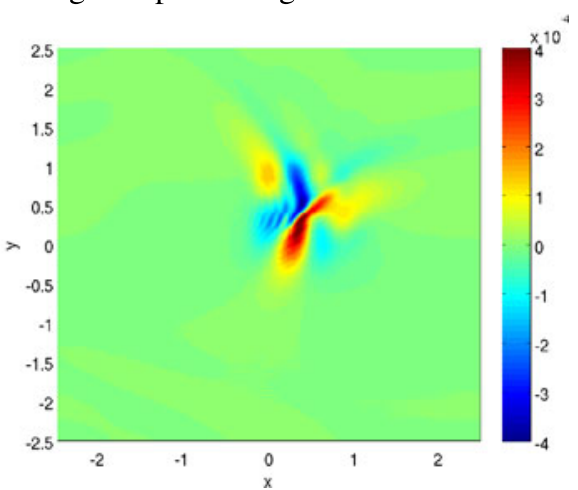

(f) $B_{3}$ component for the divergence preserving scheme.

Fig. 5 Comparison of solution with $\eta=0,5, \delta_{i}=0.2$ and $\delta_{e}=0.3162$ after $T=0.4$. On the left we have the result of the symmetric scheme, and on the right of the divergence preserving schemes 
The second step of our strategy is to approximate the full MHD equations with Hall effect. The stable discretizations of the resistivity, electron inertia and Hall term will have to couple a suitable Riemann solver based finite volume method, for instance the approximate Riemann solvers proposed in [4]. As the divergence preserving scheme was able to handle discontinuous velocities, we believe that this coupling will be robust and will enable us to design an efficient numerical scheme for the full MHD equations with Hall effect. This coupling is a part of current research and results will presented in forthcoming papers.

There are a couple of open issues with the proposed schemes-the first (as remarked before) is to design preconditioners to solve the linear equations that result in every sub-step of our time stepping routine. The second is the issue of robust boundary conditions. Both are subject to ongoing research.

\section{Appendix: Finite difference operators}

The different operators used in our numerical experiment, are based on one dimensional operators coupled together with Kronecker product. The one dimensional operators are given for $q=x, y, z$ in matrix form:

- Second order central difference

$$
D_{q}^{(2)}=P_{q}^{-1} Q=\frac{1}{2 \Delta q}\left(\begin{array}{ccccc}
-2 & 2 & & & \\
-1 & 0 & 1 & & \\
& \ddots & \ddots & \ddots & \\
& & -1 & 0 & 1 \\
& & & -2 & 2
\end{array}\right), \quad P_{q}=\Delta q\left(\begin{array}{cccc}
\frac{1}{2} & & & \\
& 1 & & \\
& & \ddots & \\
& & 1 & \\
& & & \frac{1}{2}
\end{array}\right) .
$$

- Fourth order central difference

$$
\begin{aligned}
& D_{q}^{(4)}=P_{q}^{-1} Q=\frac{1}{\Delta q}\left(\begin{array}{ccccccccc}
-1 & 1 & & & & & \\
-\frac{1}{2} & 0 & \frac{1}{2} & & & & \\
\frac{1}{12} & -\frac{2}{3} & 0 & \frac{2}{3} & -\frac{1}{12} & & \\
& \ddots & \ddots & \ddots & \ddots & \ddots & \\
& & \frac{1}{12} & -\frac{2}{3} & 0 & \frac{2}{3} & -\frac{1}{12} \\
& & & & -\frac{1}{2} & 0 & \frac{1}{2} \\
& & & & & -1 & 1
\end{array}\right) \text {, } \\
& P_{q}=\Delta q\left(\begin{array}{ccccc}
\frac{1}{2} & & & & \\
& 1 & & & \\
& & \ddots & & \\
& & & 1 & \\
& & & & \frac{1}{2}
\end{array}\right) \text {. }
\end{aligned}
$$

Combining this operators we obtain the two spatial discretisation used in the numerical experiments. 
We give the discrete derivative for the $x$ direction, the ones for the other spatial directions are defined analogously.

Standard second and fourth order operator are

$$
\mathfrak{d}_{x}=D_{x}^{(k)} \otimes I_{y} \otimes I_{z}, \quad k=2,4
$$

where $I_{q}$ are the identity matrices.

\section{References}

1. Biskamp, D.: Nonlinear Magnetohydrodynamics. Cambridge University Press, New York (1993)

2. Carpenter, M.H., Nordström, J., Gottlieb, D.: A stable and conservative interface treatment of arbitrary spatial accuracy. J. Comput. Phys. 148, 341-365 (1999)

3. Fuchs, F.G., Karlsen, K., Mishra, S., Risebro, N.H.: Stable upwind schemes for the magnetic induction equation. Math. Model. Numer. Anal. 43(5), 825-852 (2009)

4. Fuchs, F.G., McMurry, A.D., Mishra, S., Risebro, N.H., Waagan, K.: Simulating waves in the upper solar atmosphere with SURYA: a well-balanced high-order finite volume code. Astrophys. J. 732(2), 75 (2011)

5. Gottlieb, S., Shu, C.-W., Tadmor, E.: Strong stability-preserving high-order time discretisation method. SIAM Rev. 43, 89-112 (2001)

6. Gurnett, D.A., Bhattacharjee, A.: Introduction to Plasma Physics. Cambridge University Press, New York (2005)

7. Hiptmair, R.: Multi-grid method for Maxwell's equations. SIAM J. Numer. Anal. 36(1), 204-225 (1999)

8. Koley, U., Mishra, S., Risebro, N.H., Svärd, M.: Higher order finite difference schemes for the magnetic induction equations. BIT Numer. Math. 49(2), 375-395 (2009)

9. Koley, U., Mishra, S., Risebro, N.H., Svärd, M.: Higher order finite difference schemes for the magnetic induction equations with resistivity. IMA J. Numer. Anal. (2011). doi:10.1093/imanum/drg030

10. Kreiss, H.-O., Scherer, G.: Finite element and finite difference methods for hyperbolic partial differential equations. In: deBoor, C. (ed.) Mathematical Aspects of Finite Elements in Partial Differential Equations, pp. 195-212. Academic Press, New York (1974)

11. Ma, Z.W., Bhattacharjee, A.: Hall magnetohydrodynamic reconnection: the geospace environment challenge. J. Geophys. Res. 106, 3773-3782 (2001)

12. Mattsson, K., Nordström, J.: Summation by parts operators for finite difference approximations of second derivatives. J. Comput. Phys. 199, 2 (2004)

13. Mishra, S., Svärd, M.: On stability of numerical scheme via frozen coefficients and magnetic induction equations. BIT Numer. Math. 50(1), 85-108 (2010)

14. Mishra, S., Tadmor, E.: Constraint preserving schemes using potential-based fluxes. I. Multidimensional transport equations. Commun. Comput. Phys. 9(3), 688-710 (2010)

15. Qian, X., Balbás, J., Bhattacharjee, A., Yang, H.: A numerical study of magentic reconnection: A central scheme for Hall MHD. In: Tadmor, E., Liu, J.-G., Tzavaras, A. (eds.) Hyperbolic Problems: Theory, Numerics, Applications. Proceedings of the 12th International Conference held in University of Maryland, June 2008. AMS Proc. Symp. Applied Math., vol. 67, pp. 879-888 (2008)

16. Strand, B.: Summation by parts for finite difference approximations for $\mathrm{d} / \mathrm{dx}$. J. Comput. Phys. 110, 47-67 (1994)

17. Svärd, M., Nordström, J.: On the order of accuracy for difference approximations of initial-boundary value problems. J. Comput. Phys. 218, 333-352 (2006)

18. Torrilhon, M., Fey, M.: Constraint-preserving upwind methods for multidimensional advection equations. SIAM J. Numer. Anal. 42(4), 1694-1728 (2004)

19. Toth, G., Ma, Y.J., Gombosi, T.I.: Hall magnetohydrodynamics on block adaptive grids. J. Comput. Phys. 227, 6967-6984 (2008) 